\title{
Antidisturbance Vibration Suppression of the Aerial Refueling Hose during the Coupling Process
}

\author{
Zikang Su ${ }^{1,2,3}$ and Honglun Wang ${ }^{1,3}$ \\ ${ }^{1}$ School of Automation Science and Electrical Engineering, Beihang University, Beijing 100191, China \\ ${ }^{2}$ Shen Yuan Honors College of Beihang University, Beijing 100191, China \\ ${ }^{3}$ Science and Technology on Aircraft Control Laboratory, Beihang University, Beijing 100191, China
}

Correspondence should be addressed to Honglun Wang; wang_hl_12@126.com

Received 19 June 2017; Revised 12 October 2017; Accepted 17 October 2017; Published 31 December 2017

Academic Editor: Kenneth M. Sobel

Copyright (C) 2017 Zikang Su and Honglun Wang. This is an open access article distributed under the Creative Commons Attribution License, which permits unrestricted use, distribution, and reproduction in any medium, provided the original work is properly cited.

\begin{abstract}
In autonomous aerial refueling (AAR), the vibration of the flexible refueling hose caused by the receiver aircraft's excessive closure speed should be suppressed once it appears. This paper proposed an active control strategy based on the permanent magnet synchronous motor (PMSM) angular control for the timely and accurate vibration suppression of the flexible refueling hose. A nonsingular fast terminal sliding-mode (NFTSM) control scheme with adaptive extended state observer (AESO) is proposed for PMSM take-up system under multiple disturbances. The states and the "total disturbance" of the PMSM system are firstly reconstituted using the AESO under the uncertainties and measurement noise. Then, a faster sliding variable with tracking error exponential term is proposed together with a special designed reaching law to enhance the global convergence speed and precision of the controller. The proposed control scheme provides a more comprehensive solution to rapidly suppress the flexible refueling hose vibration in AAR. Compared to other methods, the scheme can suppress the flexible hose vibration more fleetly and accurately even when the system is exposed to multiple disturbances and measurement noise. Simulation results show that the proposed scheme is competitive in accuracy, global rapidity, and robustness.
\end{abstract}

\section{Introduction}

Unmanned aerial vehicles (UAVs) [1, 2] have been widely used in civilian or military fields in recent years, which include but are not limited to target tracking, combat radius increasing, flight range extending, and battle operational efficiency improving. As an effective method for increasing the endurance and range of aircraft by refueling them in flight [3], UAV autonomous aerial refueling (AAR) has attracted an increasing interest over the last decades from both theoretical and practical aspects covering aircraft control, sensor systems, and their integration [4-8]. Generally, there are two refueling methods [3]: flying boom method and probe-drogue refueling (PDR) [9-11]. And the PDR is getting to be a universal refueling equipment due to its low cost and wide operational aspects [11].
However, there have been a growing number of catastrophic failures involving the PDR $[3,12]$. Among the failures, the flexible refueling hose vibration problem during autonomous aerial refueling (AAR) coupling [13], which is the result of excess closure speed caused by the receiver pilot, plays an extremely dangerous role to the AAR safety. The closure rate of the receiver aircraft must be constrained within a required range. The refueling coupling flight will fail to latch below the minimum speed, whereas a violent reverberation through the flexible hose (namely, the hose vibration, as shown in Figure 1) can lead to equipment severe damage at a higher closure rate. When the receiver aircraft probe couples the drogue at a higher closure rate, it will definitely cause excessive slack of the refueling flexible hose as the probe pushes the drogue forward. Then, the internal hose tension will rapidly decrease, and the 


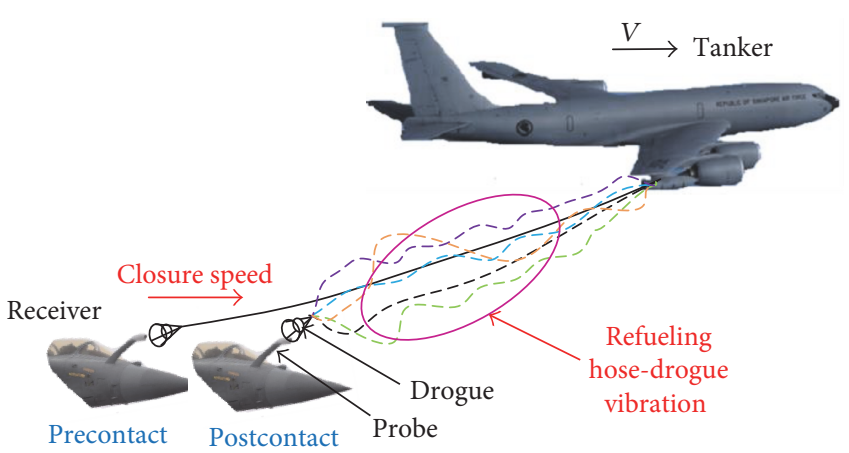

FIGURE 1: The refueling hose-drogue assembly vibration due to excessive closure speed.

hose will violently vibrate under the action of the unpredictable aerodynamic forces. Finally, tremendous high load torques on the hose and the probe rises, and equipment damage occurs. The refueling hose vibration greatly constraints the AAR's success rates and security. This drastic hose vibration requires some vibration suppression strategies to ensure the flexible refueling hose-drogue assembly stable even under unexpected excessive closure speed and multiple disturbances.

During coupling, the conventional pod is equipped with a tensator $[13,14]$ (spring-loaded take-up device) to retract any slack in the hose as the probe pushes the drogue forward. If the tensator malfunctions or being subjected to an excessive closure speed during coupling, slack in the hose can form. Then with the rapidly decreasing hose tension, the violent hose vibration occurs due to aerodynamic forces [15]. The vibration will generate high internal loads on the hose and exert high loads on the probe. While these extreme loads only exist for a fraction of a second, they result in critical damage to the refueling assembly and the receiver probe, which eventually lead to a potentially catastrophic accident. The refueling hose vibration has been a serious constraint to the success rates and security of AAR. Therefore, the vibration must be rapidly suppressed once it appears via specially designed hose take-up control system.

Although some experiments and model analysis are conducted to further research dynamic characteristics of the flexible refueling hose vibration [13-15], the conventional suppression methods do not show satisfactory performance in a more adverse condition. The Boeing Company confirmed that the reel take-up speed lagging behind the closure speed was responsible for the failure by numerical simulation [14]. That means, although every pod is equipped with a tensator to suppress the hose vibration at present, the tensator may not be the sufficient effective strategy. Alden and Vennero [16] invented a new refueling pod with a reel driven by a permanent magnet synchronous motor (PMSM), and it provides another chance for high-performance suppression methods for the vibration by integrating the PMSM and high-precision position sensors. Wang et al. [15] designed an active integral slidingmode back-stepping control strategy for the PMSM to suppress the refueling hose vibration on the basis of the relative position between the tanker and the receiver. This strategy achieves satisfactory performance on the condition when the PMSM model is precise enough and the equipment's working environment is ideal enough. However, the internal and external disturbance of the PMSM control system and the measurement noise have not been taken into consideration.

Unfortunately, during the AAR, the PMSM installed in the pod is in an extremely hostile environment [3], multiple known or unknown strong disturbances can easily disable the PMSM control system designed based on the nominal model and ideal environment. The complex working environment may be caused by the drastic vibration of the tanker's wing, the uncertain load by the hose-drogue, and other unknown uncertainty and disturbance. Meanwhile, due to the rapidly drastic changing dynamic of the flexible hose vibration, an antidisturbance rapid response control system needs to be designed for the PMSM based take-up system to suppress the hose vibration.

Although there are many existing schemes for PMSM in practical $[17,18]$, we modestly find that few works focus on the PMSM tracking control and its extended applications if the following conditions are all considered at the same time: (1) only the position sensor is equipped and the speed sensor is unavailable; (2) the position sensor measurement signal is polluted by white noise; and (3) multiple internal and external disturbances concurrently exist in the PMSM drive system [19]. It should be specially pointed out that the dynamic of PMSM is an essential nonlinear subject to a wide range of disturbances/uncertainties in many highperformance applications. According to Yang et al. [19], the disturbances/uncertainties in PMSM systems can be classified as unmodeled dynamics, parametric uncertainties, and external disturbances. The unmodeled dynamics generally include the motor body structure-induced torque (such as cogging torque, flux harmonics, and other factors), deadtime effects, and measurement error effects. Parametric uncertainties contain the uncertainties of the mechanical parameters and electrical parameters. The load torque, friction torque, and other mechanical factors are usually classified as the external disturbance. In the AAR, the load torque caused by the hose-drogue assembly is actually the primary important part of the external disturbance. In order to simplify the description and to separate from the external disturbances, we regard the unmodeled dynamics and parametric uncertainties as the internal disturbance in this paper.

However, most of the existing literatures on the PMSM controller design generally focus on the handling of either condition (1) or (3) [18-22]. The condition (2) has never been synchronously considered together with (1) and (3) in the existing works. This paper focuses on the designing of the PMSM tracking control system which satisfies the rapidity and accuracy requirements of vibration suppression and capable of handling all of those scenarios together.

In recent decades, the sliding-mode control (SMC) $[23,24]$ has been successfully applied to many uncertain systems and areas $[25,26]$, due to its superiorities such as strong 
robustness, order reduction, easier implementation, and design simplification [27]. Among the SMC methods, the terminal sliding-mode (TSM) controller has drawn much attention. It has been further developed to the nonsingular terminal sliding mode (NTSM) [27] and has been applied to many plants $[28,29]$. It has shown its fast finite time convergence, strong robustness, and preferable ability to handle the uncertainties and external disturbances. Besides, the adaptive extended state observer (AESO) [30], which possesses the excellent ability of state estimation and noise filtering, is proposed for a class of nonlinear systems with uncertain dynamics and sensor measurement noise. The gain of extended state observer (ESO) is automatically timely tuned to reduce the estimation errors of both states and "total disturbance" against the measurement noise. Most important of all, it provides another preferable way for the reconstruction of the PMSM system states and "total disturbance" on the condition when the sensor signals are polluted by noise.

With the NTSM controller and the AESO, an NTSM control scheme is proposed based on the "controller + observer" structure [24] for the PMSM tracking control with multiple disturbances (external and internal) and measurement noise. The designed control method provides another considerable choice for the problem of refueling hose vibration suppression which requires special consideration of the PMSM take-up control system's ability of the antidisturbance and rapid response. Based on those considerations, following works are investigated.

Firstly, to ensure the desired antidisturbance ability of the closed-loop system, the internal disturbances, external disturbances, and the items which are independent of the virtual control in the PMSM's speed subsystem are regarded as the "total disturbance," and then AESO is used to reconstitute the "total disturbance" and the system states in the presence of angular position measurement noise. This reconstituted "total disturbance" is further used for compensation during the controller design to improve the antidisturbance performance.

Secondly, to further improve the tracking rapidity and accuracy of the PMSM take-up system, a nonsingular fast terminal sliding-mode (NFTSM) controller with faster convergence performance is proposed for a class of uncertain nonlinear system with multiple disturbances. Some new strategies are introduced into both the sliding variable and reaching law. On one hand, a fast terminal sliding variable with tracking error exponential term is proposed to accelerate the convergence speed on the sliding surface. On the other hand, a terminal attractor with negative state exponential term is adopted for the reaching law to bring the sliding variable to the sliding surface and to eliminate the singular problem in the controller.

Thirdly, a new control scheme based on the NFTSM and AESO is designed and is well applied to the practical problem of the flexible aerial refueling hose suppression in AAR. The unexpected multiple disturbances and the measurement noise are all taken into consideration during controller design, and the angular speed sensor is needless for the feedback.
The paper is organized as follows. Section 2 describes the formulation of the PMSM tracking control problem. In Section 3 , the nonsingular terminal sliding-mode control scheme with adaptive extended state observer is proposed with the relevant proofs. Section 4 applies the proposed control scheme to the PMSM tracking control for vibration suppression with a variable length link-connected dynamic model of the hose-drogue assembly, and then, comparative simulations are conducted. Finally, conclusions are given in Section 5.

\section{Problem Formulation}

2.1. Model of the PMSM System. The accurate mathematical model of the PMSM [15,31], when there are no magnetic saturation, hysteresis, eddy current loss, or friction of the reducer, excluding sinusoidal magnetic field distribution, can be described in the $d-q$ coordinate system as

$$
\begin{aligned}
& \dot{\vartheta}=\omega, \\
& \dot{\omega}=\frac{3 P \psi_{\mathrm{f}}}{2 J} i_{q}-\frac{B}{J} \omega-\frac{T_{L}}{J}, \\
& \dot{i}_{q}=-\frac{R}{L_{\mathrm{s}}} i_{q}-P \omega i_{d}-\frac{P \psi_{\mathrm{f}}}{L_{\mathrm{s}}} \omega+\frac{u_{q}}{L_{\mathrm{s}}}, \\
& i_{d}=-\frac{R}{L_{\mathrm{s}}} i_{d}+P \omega i_{q}+\frac{u_{d}}{L_{\mathrm{s}}},
\end{aligned}
$$

where $\vartheta$ is the PMSM rotor angular position, $\omega$ is the PMSM rotor angular velocity, $i_{d}, i_{q}, u_{d}$, and $u_{q}$ are $d, q$ axis currents and voltages, respectively. $R, L_{\mathrm{s}}, P, \psi_{\mathrm{f}}, J, B$, and $T_{\mathrm{L}}$ are the stator resistance, stator inductance, the number of pole pairs, the stator flux linkage, the moment of inertia, the viscous friction coefficient of the PMSM, and the load torque, respectively.

When the internal disturbance, external disturbances, and the angular position measurement noise are considered, the PMSM's speed subsystem $S_{1}$ can be transformed into the form of

$$
S_{1}:\left\{\begin{array}{l}
\dot{\vartheta}=\omega, \\
\dot{\omega}=\frac{3 P \psi_{\mathrm{f}}}{2 J} i_{q}-\frac{B}{J} \omega-\frac{T_{\mathrm{L}}}{J}+d_{\mathrm{in}}+d_{\mathrm{ex}}=F_{1}+b_{1} i_{q}, \\
y_{1}=\theta+n_{i},
\end{array}\right.
$$

where $d_{\text {in }}$ denotes the internal disturbances, $d_{\text {ex }}$ denotes the external disturbances except $T_{\mathrm{L}} / J, y_{1}$ is the output, $n_{i}$ is the measurement noise of the position sensor, $b_{1}=$ $3 P \psi_{\mathrm{f}} / 2 J$, and $F_{1}=-(B / J) \omega-\left(T_{L} / J\right)+d_{\text {in }}+d_{\text {ex }}$ is defined as the sum of the internal disturbance, external disturbances, and the items which are independent of the virtual control in the PMSM's speed subsystem and it is taken as the total disturbances of subsystem. The disturbance $d_{1}=$ $d_{\text {in }}+d_{\text {ex }}$ satisfies $d_{1}<l_{1}$, where $l_{1}$ is a constant that is greater than zero.

Actually, the ideal model (1) always faces the challenge of many unavoidable disturbances when the PMSM is applied 


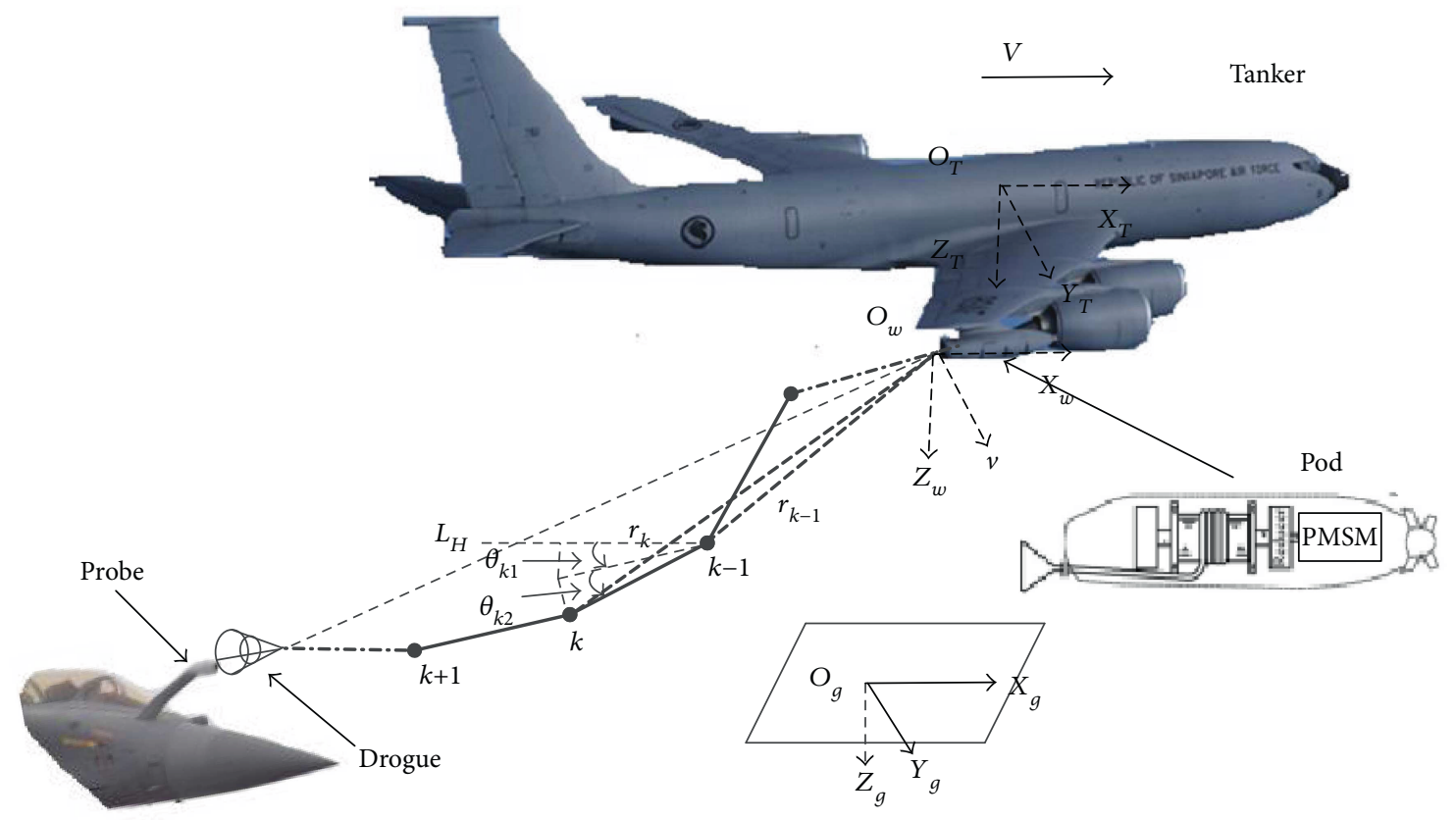

Receiver

FIGURE 2: The configuration and variable length modeling of a hose-drogue aerial refueling system.

to vibration suppression of the flexible refueling hose in AAR. Generally, the PMSM-based refueling hose-drogue reel in/out servo system which is used to suppress the vibration is equipped at the long wingtip of the tanker, as shown in Figure 2. The PMSM system faces different disturbance existing concurrently under this tough work environment. On one hand, besides the unmodeled dynamic, the PMSM system works under the integrated effect of the strong wing vibration and strong electromagnetic interference. These may provoke the strong and fast-changing parametric uncertainties (belong to the internal disturbances according to above definition). On the other hand, the unpredictable dynamics of the hose-drogue assembly and the unpredictable vibrational states of the tanker wing will definitely lead to the unpredictable and fast-changing load torque and friction torque (belong to the external disturbances). Moreover, the demanding work environment will generally make the measurement noise of the sensors inevitable. According to (2), the total disturbances $F_{1}$ will directly affect the dynamics of the speed subsystem $S_{1}$, and the measurement noise $n_{i}$ will definitely affect the closed-loop performance via the feedback channel. Ultimately, the total disturbances and the measurement noise will definitely impair the closed-loop performance. Thus, these disturbances and measurement noise should be considered during the controller design.

Generally, the two loop scheme $[18,21]$ (current loop and speed loop) is usually employed during the PMSM controller design due to different features and required qualities of these two control loops. Actually, the mechanic inertia of the speed loop is greater than that of the current loop. However, the electric inertia of the current loop is very small and it requires fast electromagnetic torque response, which makes its sampling frequency very high. Hence, the current control loop algorithm is usually desired to be as simple as the proportion integral (PI) controller or the proportion integral derivative (PID) controller [18]. Therefore, the controllers usually employ a structure of cascade control loops including a speed loop and two current loops. Here, two PI controllers are adopted in the two current loops to stabilize the current tracking errors of $d-q$ axes, respectively. Usually, the reference $i_{d}{ }^{*}$ in the $\mathrm{d}$ current loop is set to be zero to maintain a constant flux operating condition. The reference current $i_{q}^{*}$ is determined by $S_{1}$.

2.2. Command Transformation for the Vibration Suppression of Refueling Hose. In the AAR, it is assumed that the reel is driven by a PMSM to deploy or retrieve the hose through a reducer. A probe-drogue refueling system includes a reel mechanism, a refueling hose, and a drogue shown in Figure 2. A set of iterative equations of the hose's threedimensional motion is derived subject to hose reeling in/ out, tanker motion, gravity, and aerodynamic loads accounting for the effects of steady wind, atmospheric turbulence, and tanker vortex. The details of the model of the hosedrogue assembly can be found in [15].

Considering the mechanical inertia [15], the load torque $T_{\mathrm{L}}$ can be written as the transfer function of the first link hose tension $T_{1}$ of the hose-drogue assembly: $T_{\mathrm{L}}=(r / i)(1 /(s+1))$ $T_{1}$, where $r$ is the radius of the reel and $i$ is the reduction ratio of the reducer.

The most effective way to suppress the violate vibration of the refueling hose is to stabilize the hose tension by realtime active hose reeling in/out based on the relative position between the tanker and the receiver. The refueling hose 


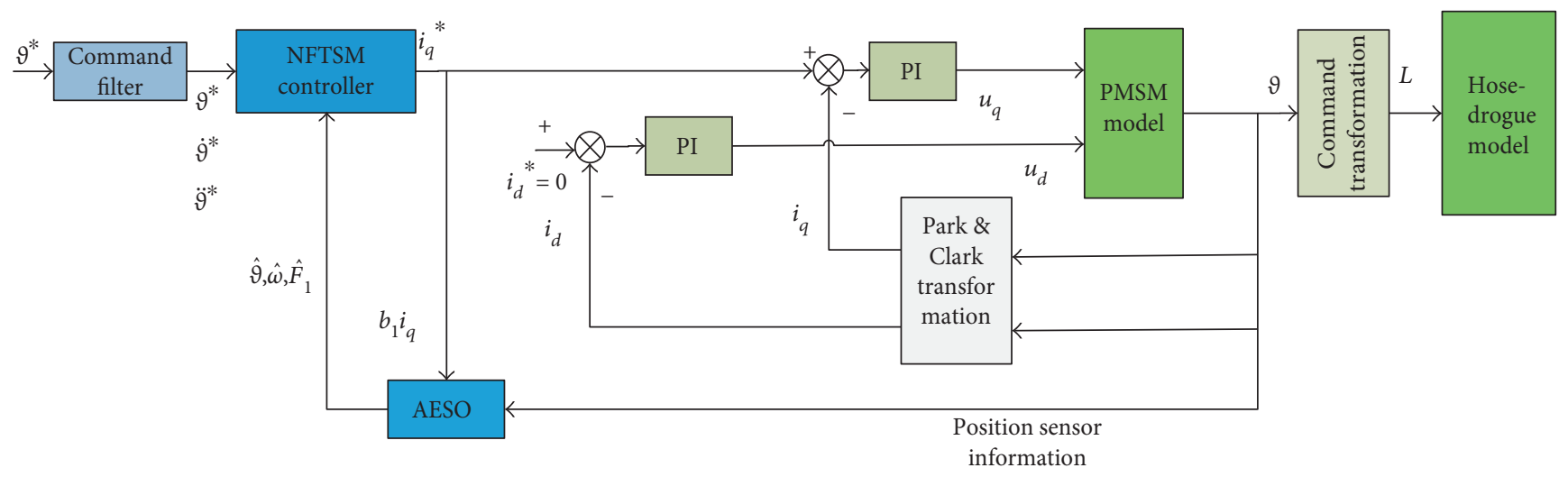

FIGURE 3: Block diagram of the PMSM control system for refueling hose vibration suppression in AAR.

length change $L$ can be expressed as a function of the angular position $\vartheta$ of PMSM as follows:

$$
L=\frac{\vartheta r}{i}
$$

where $L$ is also a function of the relative distance between the probe and the pod during coupling, which is calculated as

$$
L=\lambda \sqrt{\Delta x^{2}+\Delta y^{2}+\Delta z^{2}}-L_{0}
$$

where $\lambda=L_{0} / L_{H}$, as shown in Figure 2, reflects the degree of hose slack. $L_{0}$ is the initial length of the hose before coupling, and $\Delta x, \Delta y$, and $\Delta z$ are three spatial components of the relative distance between probe and pod [16], which can be accurately measured by three position sensors, respectively, installed on the tanker, receiver, and drogue. Therefore, the length control of the hose (hose reeling in/out) can be transformed into the rotor angular position control of the PMSM through (1).

As the vibration suppression dynamic process which is decided by the excessive closure speed of the receiver aircraft during coupling is very short (usually, lasts only about $1 \mathrm{sec}-$ ond to 2 seconds [13]), the control schemes which cannot work timely and rapidly will result in the shake of the hosedrogue becoming longer and more intense. During the hose vibration suppression process, one expects the fluctuations of the load and the hose to be as stable as possible, and this expectation can be achieved by designing a control scheme that can track the angular command rapidly and timely. Consequently, the task here is to design a controller for the speed loop that tracks the given angular signal (which is transformed from the relative distance between the drogue and the end of the pod after successful coupling) as soon as possible under the condition that various disturbances and measurement noise of the sensors exist.

\section{The Nonsingular Fast Terminal Sliding- Mode (NFTSM) Control Scheme with Adaptive Extended State Observer (AESO)}

As assumed in the introduction, the speed sensor is unavailable and measurement noise exists in the position sensor; hence, the states and disturbance should be reconstructed during the control system design. The recently proposed that AESO provides a befitting choice to reconstruct states and disturbance of the PMSM with measurement noise. With the reconstructed system by AESO, a new nonsingular fast terminal sliding-mode control method, which not only accelerates the error convergence but also reduces the overshoot, is proposed here. The structure of the proposed control scheme is depicted in Figure 3.

3.1. System Reconstitution via AESO. The adaptive extended state observer (AESO) is proposed for the nonlinear systems with the uncertainties both in the plant and in the sensor [30]. The gain of ESO is automatically timely tuned to reduce the estimation errors of both states and "total disturbance" against the measurement noise. The theory of AESO can be briefly described as follows [30].

Consider the following class of nonlinear uncertain continuous systems with measurement noise.

$$
\begin{aligned}
& \dot{X}(t)=A X(t)+B F(X(t), t)+B B_{u} U(i h), \\
& Y(i h)=X_{1}(i h)+n_{i}, \quad t \in[i h, i h+h), i=0,1, \ldots,
\end{aligned}
$$

where

$$
\begin{aligned}
& A=\left[\begin{array}{cccc}
0 & I & \cdots & 0 \\
\cdots & \cdots & \cdots & 0 \\
0 & 0 & \cdots & I \\
0 & 0 & \cdots & 0
\end{array}\right]_{m n \times m n}, \\
& B=\left[\begin{array}{c}
0 \\
0 \\
\cdots \\
I
\end{array}\right]_{m n \times m}
\end{aligned}
$$


$X=\left[\begin{array}{lllll}X_{1}{ }^{T} & X_{2}{ }^{T} & \cdots & X_{n-1}{ }^{T} & X_{n}{ }^{T}\end{array}\right]^{T}, \quad X_{j}^{T} \in R^{m}, \quad j \in \underline{n}=\{1,2$, $\ldots, n\}$ are continuous state vectors. $X_{1}(t)$ is the output to be controlled, $Y(i h) \in R^{m}$ is the measured output which contains the measurement noise vector $n_{i} \in R^{m}$, and $h>0$ is the sampling period. $B_{u}$ is a known nonsingular matrix and the control input $U(t)$ implemented by the zero-hold-device, that is, $U(t) \equiv U(i h)$ for $t \in[i h, i h+h)$. In addition, it is important to remark that $F(X(t), t) \epsilon$ $R^{m}$ in (5) includes both uncertain internal dynamics and external disturbances in the system (5), namely, the "total disturbance."

Remark 1. There are some assumptions for the system (5) that should be declared:

(A1) $\left\{n_{i}\right\}_{0}^{\infty}$ is a white random sequence and $E\left(n_{i} n_{i}^{T}\right)$ $\leq \bar{R}$, where $\bar{R}$ is a known matrix.

(A2) $E\left[\begin{array}{c}X(0)-\widehat{X}(0) \\ F(0)-\widehat{F}(0)\end{array}\right]\left[\begin{array}{c}X(0)-\widehat{X}(0) \\ F(0)-\widehat{F}(0)\end{array}\right]^{T} \leq P_{0}$, where $\widehat{X}$ and $\widehat{F}$ are the estimation of $X$ and $F$ and $P_{0}$ is a known matrix.

(A3) $(F(X(t), t)-F(X(i h), i h))(F(X(t), t)-F(X(i h), i h))^{T}$ $\leq \bar{Q}$, where $t \in[i h, i h+1)$ and $\bar{Q}$ is a known diagonal matrix.

A1-A2, which essentially assume the noise and the initial estimation errors to be bounded in the mean square, are reasonable due to the bounds of the sensor noise in practice. A3 implies that the varying of the uncertainty function $F(\cdot)$ in one sampling period is bounded.

The goal of the AESO is to get the precise estimations of the states $X_{j}^{T}, \quad j=\{1,2, \ldots, n\}$ and the total disturbance $F(X(t), t)$ despite the existence of various kinds of uncertainties and measurement noise, as the engineers usually desire. These precise estimations can be used to design the control input for the PMSM.

Note that the discrete error is usually decided by the sampling rate which is fixed by the hardware and the estimation error $\xi(i h)=[X(i h)-\widehat{X}(i h) F(i h)-\widehat{F}(i h)]^{T}$. Lemma 1 shows the formulas and the properties of the estimation error $\xi(i h)$.

Lemma 1 [30]. Let A1-A3 hold, and the AESO for the general integral serial system is given as

$$
\begin{aligned}
{\left[\begin{array}{c}
\widehat{X}(i h+h) \\
\widehat{F}(i h+h)
\end{array}\right]=} & \bar{A}\left[\begin{array}{l}
\widehat{X}(i h) \\
\widehat{F}(i h)
\end{array}\right]+\widehat{L}_{i}\left(Y(i h)-\widehat{X}_{1}(i h)\right) \\
& +\left[\begin{array}{c}
\bar{B} B_{u} U(i h) \\
0
\end{array}\right] \\
\widehat{L}_{i} & =\bar{A} P_{i} C\left(C^{T} P_{i} C+\frac{1}{1+\theta} \bar{R}\right)^{-1}
\end{aligned}
$$

$$
\begin{aligned}
P_{i+1}= & (1+\theta)\left(\bar{A}-\bar{L}_{i} C^{T}\right) P_{i}\left(\bar{A}-\bar{L}_{i} C^{T}\right)^{T} \\
& +\bar{L}_{i} \bar{R} \bar{L}_{i}^{T}+\left(1+\frac{1}{\theta}\right) Q .
\end{aligned}
$$

Moreover, the estimation errors $\{\xi(i h)\}_{i=0}^{\infty}$ are uniformly bounded in the mean square and $E\left(\xi(i h) \xi(i h)^{T}\right) \leq P_{i}, \quad \forall i \geq$ 0 . There exists a positive definite matrix $P^{*}$ so that $\lim P_{i}=$ $P^{*}$, where

$\bar{A}=\exp \left(\left[\begin{array}{ll}A & B \\ 0 & 0\end{array}\right] h\right)=\left[\begin{array}{cccc}I & \frac{h^{1}}{1 !} I & \ldots & \frac{h^{n}}{n !} I \\ 0 & I & \cdots & \left.\frac{h^{n-1}}{(n-1) !} I\right], \\ \ldots & \ldots & \ldots & \ldots \\ 0 & 0 & \cdots & I\end{array}\right]$,
$\bar{B}=\int_{0}^{h} \exp \left(\left[\begin{array}{ll}A & B \\ 0 & 0\end{array}\right](h-\tau)\right) d \tau\left[\begin{array}{c}\frac{h^{n}}{n !} I \\ 0\end{array}\right]=\left[\begin{array}{c}\frac{h^{n-1}}{(n-1) !} I \\ \cdots \\ h I\end{array}\right]$,

$Q=m(n+1)\left[\begin{array}{cccc}h^{2 n} \bar{Q} & 0 & \cdots & 0 \\ 0 & h^{2(n-1)} \bar{Q} & \cdots & 0 \\ \cdots & \cdots & \cdots & 0 \\ 0 & 0 & \cdots & \bar{Q}\end{array}\right]$,

$C=\left[\begin{array}{l}I \\ 0 \\ \cdots \\ 0\end{array}\right]$

$\theta=\sqrt{\frac{\sum_{j=1}^{(n+1) m} Q(j, j)}{\sum_{j=1} \mathbf{P}_{0}(j, j)}}$.

Consequently, for the model (2) of $S_{1}$ with measurement noise used in AAR can be rewritten as

$$
\begin{aligned}
{\left[\begin{array}{l}
\dot{\vartheta}(t) \\
\dot{\omega}(t)
\end{array}\right]=} & {\left[\begin{array}{ll}
0 & 1 \\
0 & 0
\end{array}\right]\left[\begin{array}{l}
\vartheta(t) \\
\omega(t)
\end{array}\right]+\left[\begin{array}{l}
0 \\
1
\end{array}\right] F_{1}(t)+\left[\begin{array}{l}
0 \\
1
\end{array}\right] b_{1} i_{q}(i h), } \\
Y(i h) & =\vartheta(i h)+n_{i}, \\
& t \in[i h, i h+h), i=0,1, \ldots
\end{aligned}
$$

The PMSM system can be reconstituted via the nominal AESO (12), according to (7), (8), and (9). 


$$
\begin{aligned}
& S_{1}: \bar{A}_{1}= {\left[\begin{array}{lll}
1 & \frac{h^{1}}{1 !} & \frac{h^{2}}{2 !} \\
0 & 1 & \frac{h^{1}}{1 !} \\
0 & 0 & 0
\end{array}\right], } \\
& \bar{B}_{1}= {\left[\begin{array}{l}
\frac{h^{2}}{2 !} \\
h^{1} \\
1 ! \\
0
\end{array}\right], } \\
& \bar{C}_{1}= {\left[\begin{array}{l}
1 \\
0 \\
0
\end{array}\right], } \\
& Q_{1}=3 \bar{Q}_{1}\left[\begin{array}{ccc}
h^{4} & 0 & 0 \\
0 & h^{2} & 0 \\
0 & 0 & 1
\end{array}\right] .
\end{aligned}
$$

With the AESO (12), the system states $\vartheta, \omega$ and the total disturbance $F_{1}$ can be well estimated even with the output measurement noises $n_{i}$.

3.2. The Nonsingular Fast Terminal Sliding-Mode (NFTSM) Control. This section focuses on the rapid controller design of the PMSM take-up system based on a nonsingular terminal sliding model. Here, a new NFTSM surface and the sliding mode reaching law are given. Then, the NFTSM controller is designed for the speed loop and some of its properties are analyzed.

The conventional NTSM control is proposed to solve the controller singularity problem and improve the system tracking precision. For (2), the conventional NTSM is described by following nonlinear switching surface [27]:

$$
s_{0}=e_{1}+\frac{1}{\beta} e_{2}^{p / q},
$$

where $e_{1}=\vartheta-\vartheta^{*}, \quad e_{2}=\dot{e}_{1}=\dot{\vartheta}-\dot{\vartheta}^{*}=\omega-\dot{\vartheta}^{*}, \quad \beta>0$ is the designed parameter, and $p, q$ are the positive odd integers satisfying $1<p / q<2$.

Remark 2. When the sliding mode $s_{0}=e_{1}+(1 / \beta) e_{2}{ }^{p / q}=0$ is reached, it will reach the equilibrium within the finite time

$$
T_{n}=\int_{0}^{V_{n}(0)}\left(2^{(p+q) / 2 q} \beta V_{n}^{(p+q) / 2 q}\right)^{-p / q} d V_{n}
$$

where $V_{n}=0.5 e_{1}^{2}$ and the derivational process of (14) is given in the appendix.

For system (2) with NTSM (13), the controller can be designed as

$$
u=-b_{1}^{-1}\left[F_{1}+\beta \frac{q}{p} e_{2}^{2-p / q}+\left(l_{1}+\eta_{1}\right) \operatorname{sgn}(s)-\ddot{\vartheta}^{*}\right],
$$

where $\eta_{1}>0$ is the switching gain and $\vartheta^{*}$ is the angular position command signal to be tracked. And the NTSM surface function (15) will be reached in the finite time; furthermore, the state errors $e_{1}$ and $e_{2}$ will converge to zero.

However, for the NTSM controller (15), in order to achieve system convergence, the switching gain $\eta_{1}$ is generally selected for a bigger value [27]. It will obviously produce the chattering and influence the convergence precision during the steady state, and the chattering will further make the system unstable. From above analysis, it is very necessary to improve system convergence characteristics and eliminate system chattering based on the conventional NTSM controller.

The proposed NFTSM is described as (16), the state tracking error power item $(1 / \alpha) e_{1}{ }^{a / b}$ and the exponential term $(1 / \eta)\left|e_{1}\right| \exp \left(\left|e_{1}\right|\right)$ to improve system convergence speed in the NFTSM.

$$
s=e_{1}+\frac{1}{\alpha} e_{1}^{a / b}+\frac{1}{\beta} e_{2}^{p / q}+\frac{1}{\eta}\left|e_{1}\right| \exp \left(\left|e_{1}\right|\right)
$$

where $a, b$ are the positive odd integers satisfying $a / b>p / q$.

Remark 3. For the sliding variable (16), when the states are far from the equilibrium point, the teams $e_{1}$ and $(1 / \eta)\left|e_{1}\right|$ $\exp \left(\left|e_{1}\right|\right)$ mainly affect the convergence efficiency, which can make the system trajectory converge quickly. When the states are near to the equilibrium point, the team $(1 / \alpha) e_{1}{ }^{a / b}$ largely affects the convergence efficiency, and the $(1 / \eta)\left|e_{1}\right|$ $\exp \left(\left|e_{1}\right|\right)$ can still make the system trajectory converge quickly. So the proposed NFTSM scheme can achieve the global convergence quickly, not just within the area adjacent to the equilibrium point.

Besides the sliding surface, the reaching law is also needed to bring the sliding variable to the sliding surface. Actually, the terminal attractor, which is widely used in many sliding-mode control methods, makes the system states approach the sliding surface in a finite time and improves the robustness to uncertainties and disturbances. An effective terminal attractor-based reaching law is proposed as

$$
\dot{s}=-\phi s-\gamma s^{m / n} .
$$

However, if we use (17) as the reaching law for the system (2) with the sliding surface (16), the item $e_{2}^{1-p / q}$ will arise in the control input. As $1<p / q<2$, thus $1-p / q<0$ and $e_{2}^{1-p / q}$ will case the singular problem. To eliminate $e_{2}^{1-p / q}$ from the control input, we design a modified reaching law with terminal attractor on the basis of (17) as

$$
\dot{s}=\left(-\phi s-\gamma s^{m / n}\right) e_{2}^{p / q-1},
$$

where $\phi>0, \gamma>0$ and $m, n$ are the positive odd integers satisfying $0<m / n<1$. And in Theorem 3 , it can be found 
that the control input is absolutely nonsingular with the modified reaching law (18).

Remark 4. For the reaching law (18), $-\phi$ s plays the leading role to guarantee the faster-reaching speed primarily. When the system is near the sliding surface, $-\gamma s^{m / n}$ plays the leading role to reduce the reaching speed that makes the system states reach the sliding surface smoothly.

Based on (2), (16), and (18), then we design the NFTSM controller:

$$
\begin{aligned}
u=-b_{1}{ }^{-1} & {\left[\beta \frac{q}{p}\left(\phi s+\gamma s^{m / n}\right)+\beta \frac{q}{p} e_{2}^{2-p / q}\right.} \\
& \cdot\left(1+\frac{a}{\alpha b} e_{1}^{a / b-1}+\frac{1}{\eta} \exp \left(\left|e_{1}\right|\right)\left(1+\left|e_{1}\right|\right)\right) \\
+ & \left.\left(F_{1}-\ddot{\vartheta}^{*}\right)\right] .
\end{aligned}
$$

As $p, q, a, b, m$, and $n$ are all positive odd numbers and $1<p / q<2, p / q<a / b$, and $0<m / n<1$, thus, $2-p / q>1$ and $a / b-1>0$. Then, we know that the exponential terms of the errors $e_{1}, e_{2}$ are all positive, and no negative exponential terms appear in the controller (19). That is, no singular problem will not appear in the controller by the special designed reaching law.

The convergence characteristic of the closed-loop system is generally decided by the sliding surface and the reaching law. When the system is on the sliding phase $(s=0)$, the convergence characteristic is decided by the sliding surface; when the system is on the sliding phase, the convergence characteristic is decided by the reaching law.

Theorem 1 shows the convergence characteristic and the finite convergence time of the system (2) on the sliding surface $s=0$. And Theorem 2 shows the convergence characteristic of the reaching phase.

Theorem 1. For system (2) with the NFTSM (16), when the sliding mode $s=0$ is reached, the system states will reach the equilibrium along the sliding surface $s=0$ within the time $T_{n f}$ and $T_{n f}<T_{n}$ is satisfied, where

$$
\begin{aligned}
T_{n f}=\int_{0}^{V_{n}(0)} & \left(\tau_{1} V_{n}^{(p+q) / 2 q}+\tau_{2} V_{n}^{(a / 2 b)+(p / 2 q)}\right. \\
& \left.+\tau_{3} V_{n}^{p / 2 q}\left|V_{n}^{1 / 2}\right| \exp \left(\left|V_{n}^{1 / 2}\right|\right)\right)^{-q / p} d V_{n}
\end{aligned}
$$

Proof. When the system errors $\left(e_{1}, e_{2}\right)$ reach the sliding surface $s=0$ and with (16), we get that the system dynamics is determined by the following nonlinear differential equation:

$$
s=e_{1}+\frac{1}{\alpha} e_{1}^{a / b}+\frac{1}{\beta} e_{2}^{p / q}+\frac{1}{\eta}\left|e_{1}\right| \exp \left(\left|e_{1}\right|\right)=0,
$$

as $e_{2}=\dot{e}_{1}=\dot{\vartheta}-\dot{\vartheta}^{*}=\omega-\dot{\vartheta}^{*}$, that is,

$$
e_{2}^{p / q}=\dot{e}_{1}^{p / q}=-\beta\left(e_{1}+\frac{1}{\alpha} e_{1}^{a / b}+\frac{1}{\eta}\left|e_{1}\right| \exp \left(\left|e_{1}\right|\right)\right) .
$$

Define $V_{n}=0.5 e_{1}^{2}$; it can be got that $\dot{V}_{n}=e_{1} \dot{e}_{1}$ and then

$$
\begin{aligned}
\dot{V}_{n}^{p / q} & =e_{1}^{p / q} \dot{e}_{1}^{p / q}=-\beta e_{1}^{p / q}\left(e_{1}+\frac{1}{\alpha} e_{1}^{a / b}+\frac{1}{\eta}\left|e_{1}\right| \exp \left(\left|e_{1}\right|\right)\right) \\
& =-\beta\left(e_{1}^{p+q / q}+\frac{1}{\alpha} e_{1}^{(a / b)+(p / q)}+\frac{1}{\eta} e_{1}^{p / q}\left|e_{1}\right| \exp \left(\left|e_{1}\right|\right)\right) .
\end{aligned}
$$

As $p, q, a, b$ are all positive odd numbers; hence, $V_{n}<0$ is satisfied when $e_{1} \neq 0$. Based on the Lyapunov's stability criterion, the dynamic $s=e_{1}+(1 / \alpha) e_{1}^{a / b}+(1 / \beta) e_{2}^{p / q}+(1 / \eta)\left|e_{1}\right|$ $\exp \left(\left|e_{1}\right|\right)=0$ is stable and the system states will converge to the equilibrium. And from (22), it can be known that the only equilibrium is $e_{1}=0$. To get the convergence time $T_{n f}$, we submit $e_{1}^{2}=2 V_{n}$ into (23) and get

$$
\begin{aligned}
\dot{V}_{n}^{p / q}=- & \left(\tau_{1} V_{n}^{(p+q) / 2 q}+\tau_{2} V_{n}^{(a / 2 b)+(p / 2 q)}\right. \\
& \left.+\tau_{3} V_{n}^{p / 2 q}\left|V_{n}^{1 / 2}\right| \exp \left(\left|V_{n}^{1 / 2}\right|\right)\right),
\end{aligned}
$$

where $\tau_{1}=2^{(p+q) / 2 q} \beta, \tau_{1}=2^{(a / 2 b)+(p / 2 q)}(\beta / \alpha), \tau_{1}=2^{p / 2 q}(\beta / \eta)$.

As $\dot{V}_{n}=\left(d V_{n}\right) /(d t)$, the following can be obtained:

$$
\begin{aligned}
d t= & \frac{d V_{n}}{\dot{V}_{n}}=\frac{d V_{n}}{-\left(-\dot{V}_{n}\right)}=-\left(\left(-\dot{V}_{n}\right)^{p / q}\right)^{-q / p} d V_{n} \\
=- & \left(\tau_{1} V_{n}^{(p+q) / 2 q}+\tau_{2} V_{n}^{(a / 2 b)+(p / 2 q)}\right. \\
& \left.\quad+\tau_{3} V_{n}^{p / 2 q}\left|V_{n}^{1 / 2}\right| \exp \left(\left|V_{n}^{1 / 2}\right|\right)\right)^{-q / p} d V_{n} .
\end{aligned}
$$

Furthermore, as $V_{n}\left(T_{n f}\right)=0$ and $V_{n}(0)=0.5 e_{1}^{2}(0) \geq 0$, we integrate (25) from both sides and get the time $T_{n f}$.

$$
\begin{aligned}
& T_{n f}=\int_{0}^{V_{n}(0)}\left(\tau_{1} V_{n}^{(p+q) / 2 q}+\tau_{2} V_{n}^{(a / 2 b)+(p / 2 q)}\right. \\
& \left.+\tau_{3} V_{n}^{p / 2 q}\left|V_{n}^{1 / 2}\right| \exp \left(\left|V_{n}^{1 / 2}\right|\right)\right)^{-q / p} d V_{n}, \\
& T_{n f}=\int_{0}^{V_{n}(0)}\left(\tau_{1} V_{n}^{(p+q) / 2 q}+\tau_{2} V_{n}^{(a / 2 b)+(p / 2 q)}\right. \\
& \left.+\tau_{3} V_{n}^{p / 2 q}\left|V_{n}^{1 / 2}\right| \exp \left(\left|V_{n}^{1 / 2}\right|\right)\right)^{-q / p} d V_{n}<T_{n} \\
& =\int_{0}^{V_{n}(0)}\left(\tau_{1} V_{n}^{(p+q) / 2 q}\right)^{-q / p} d V_{n} \text {. }
\end{aligned}
$$

Remark 5. From the proof above, it can also be known that the composition of the term $(1 / \alpha) e_{1}^{a / b}$ and $(1 / \eta)\left|e_{1}\right| \exp \left(\left|e_{1}\right|\right)$ is better than the choice of single $(1 / \alpha) e_{1}^{a / b}$. Assume that the sliding variable is selected as $s_{1}=e_{1}+(1 / \alpha) e_{1}^{a / b}+(1 / \beta) e_{2}^{p / q}$, and then we can easily get that $T_{n f}<T_{n}=\int_{0}^{V_{n}(0)}$ $\left(\tau_{1} V_{n}^{(p+q) / 2 q}+\tau_{2} V_{n}^{(a / 2 b)+(p / 2 q)}\right)^{-q / p} d V_{n}$. Hence, (16) provides another good choice to obtain a faster convergence characteristic without increasing the gains in the sliding variable.

And Theorem 2 shows that the attractor in (18) will not affect the existence of the NFTSM (16) during the reaching 
phase. Moreover, the sliding mode $s=0$ will be reached asymptotically.

Theorem 2. For the system (2), if the closed-loop controller is designed as (19), then the NFTSM (16) exists globally and the system states from any initial positions will asymptotically converge to the sliding surface $s=0$.

Proof. Consider the following Lyapunov function:

$$
V_{0}=0.5 s^{2}
$$

Hence,

$$
\dot{V}_{0}=\dot{s}=\left(-\phi s^{2}-\gamma s^{m / n+1}\right) e_{2}^{p / q-1} .
$$

Define $\mathbf{e}=\left(e_{1}, e_{2}\right)$, then we divide the $e_{1}-e_{2}$ phase plane into two regions: $D_{1}=\left\{\mathbf{e} \mid e_{1} \neq 0, e_{2} \neq 0\right\}$ and $D_{2}=\left\{\mathbf{e} \mid e_{1} \neq 0\right.$, $\left.e_{2}=0\right\}$. Indeed, it should be noted that $D_{2}$ is the axis $e_{1}$ in the $e_{1}-e_{2}$ phase plane.

Suppose $l\left(e_{2}\right)=e_{2}{ }^{p l q-1}$, then $l\left(e_{2}\right)>0$ when $e_{2} \neq 0$. Namely, when $\mathbf{e}=\left(e_{1}, e_{2}\right) \in D_{1}$, we get $l\left(e_{2}\right)>0, \quad s^{m / n+1}>0$ and

$$
\dot{V}_{0}=s \dot{s}=\left(-\phi s^{2}-\gamma s^{m / n+1}\right) l\left(e_{2}\right) \leq 0 .
$$

Thus, the sliding mode $s$ will asymptotically converge to zero in the region $D_{1}$, according to Lyapunov stability criterion. That is, the NFTSM (16) exists in the region $D_{1}$.

Next, we will check whether the sliding mode $s$ will still converge to zero in $D_{2}$. Indeed, substituting the control law (19) into the error dynamic equation of (2) yields

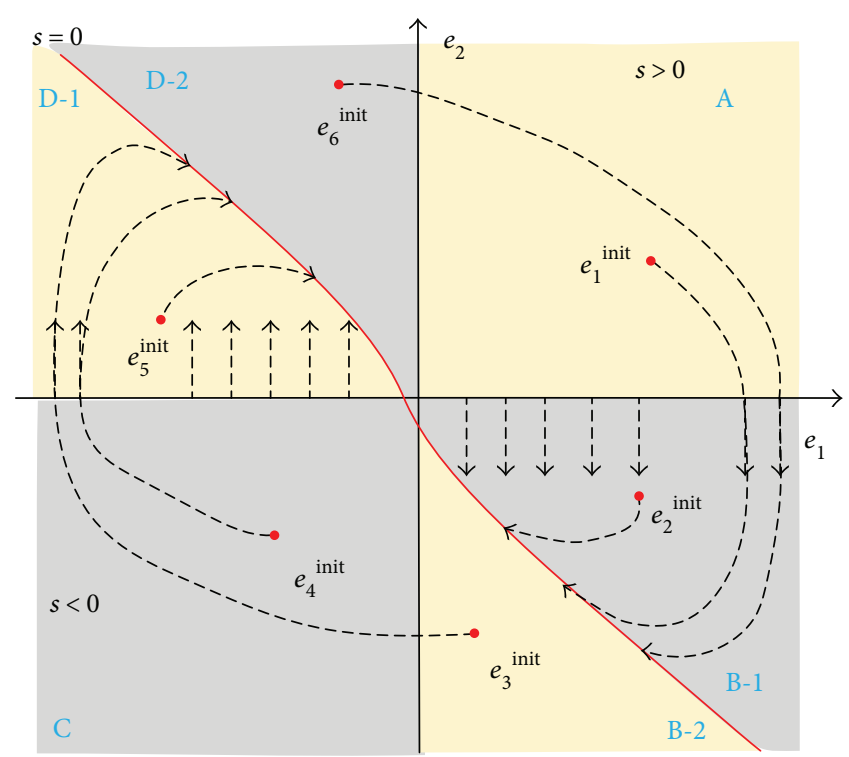

Figure 4: The relative coupling profile.

$$
\begin{aligned}
\dot{e}_{1}= & e_{2}, \\
\dot{e}_{2}= & F_{1}-\ddot{\vartheta}^{*}+b_{1} i_{q}=-\beta \frac{q}{p} \\
& \cdot\left[\left(\phi s+\gamma s^{m / n}\right)+e_{2}^{2-p / q}\left(1+\frac{a}{\alpha b} e_{1}^{a / b-1}\right.\right. \\
& \left.\left.+\frac{1}{\eta} \exp \left(\left|e_{1}\right|\right)\left(1+\left|e_{1}\right|\right)\right)\right] .
\end{aligned}
$$

From (30), we get the slope equation of the phase path.

$$
\frac{\mathrm{d} e_{2}}{\mathrm{~d} e_{1}}=\frac{\dot{e}_{2}}{\dot{e}_{1}}=\frac{-\beta(q / p)\left[\left(\phi s+\gamma s^{m / n}\right)+e_{2}^{2-p / q}\left(1+(a / \alpha b) e_{1}^{a / b-1}+(1 / \eta) \exp \left(\left|e_{1}\right|\right)\left(1+\left|e_{1}\right|\right)\right)\right]}{e_{2}} .
$$

As $p, q, a, b, m$, and $n$ are all positive odd numbers and $1<p / q<2$, and $a / b>1$, thus we get (32) when $e_{1}>0$ and $e_{2}=0$.

$$
\begin{aligned}
\frac{\mathrm{d} e_{2}}{\mathrm{~d} e_{1}}=-\infty & \\
\dot{e}_{2}=-\beta \frac{q}{p} & {\left[\left(\phi s+\gamma s^{m / n}\right)+e_{2}^{2-p / q}\right.} \\
& \left.\cdot\left(1+\frac{a}{\alpha b} e_{1}^{a / b-1}+\frac{1}{\eta} \exp \left(\left|e_{1}\right|\right)\left(1+\left|e_{1}\right|\right)\right)\right]<0 .
\end{aligned}
$$

Equation (32) means that the phase path is perpendicular to the positive axis $e_{1}$ when it crosses the positive axis $e_{1}$, and phase path direction is straight down from the positive axis $e_{1}$, as shown in Figure 4. Moreover, the motion speed of $e=\left(e_{1}, e_{2}\right)$ is not equal to zero. Similarly, the phase path is perpendicular to the negative axis $e_{1}$ when it crosses the negative axis $e_{1}$; the phase path direction is straight up, and the motion speed of $e=\left(e_{1}, e_{2}\right)$ is also not equal to zero.

The state trajectory in the reaching phase is shown in Figure 4. $e_{i}^{\text {init }}=\left(e_{1}, e_{2}\right)\left(e_{1}, e_{2}\right)_{i}^{\text {init }}, i=1, \ldots, 6$ are the possible locations for the system initial states throughout the whole phase plane. The region $D_{1}$ is then separated into six regions (A, B-1, B-2, C, D-1, and D-2) to illustrate the state trajectory of any possible initial states during the reaching phase. Indeed, as concluded above, the system state's movement speed is not zero when it crosses the axis $e_{1}$ (namely, the system state is in $D_{2}$ ). Therefore, although the state trajectory may cross the axis $e_{1}$ (such as $\mathbf{e}_{i}^{\text {init }}, \quad i=1,3,4,6$ ), the state $\mathbf{e}$ will not stay in $D_{2}$. In these cases, the states $\mathbf{e}_{i}^{\text {init }}, \quad i=1,3,4,6$ will cross the axis $e_{1}$. Then, they will asymptotically converge to the sliding mode $s=0$ like the initial states which do not cross axis $e_{1}$ (such as, $\mathbf{e}_{i}^{\text {init }}, i=2,5$ ). 
3.3. The Proposed Control Scheme for PMSM Tracking Control. Based on the design and analysis of the AESO and NFTSM above, Theorem 3 gives the proposed controller for the PMSM angular position tracking control to suppress the hose vibration problem in AAR coupling.

Theorem 3. Under Assumptions A1-A3, for the system (2) with the NFTSM (16), if the AESO is designed with (7), (8), and (9) and the closed-loop controller is designed as (33), then the system trajectory will fast converge to zero. Moreover, no occurring of singularity is ensured during the whole process.

$$
\begin{array}{r}
u=-b_{1}^{-1}\left[\beta \frac{q}{p}\left(\phi \widehat{s}+\gamma \widehat{s}^{m / n}\right)+\left(\widehat{F}_{1}-\ddot{\vartheta}^{*}\right)+\beta \frac{q}{p} \widehat{e}_{2}{ }^{2-p / q}\right. \\
\left.\cdot\left(1+\frac{a}{\alpha b} \widehat{e}_{1}^{a / b-1}+\frac{1}{\eta} \exp \left(\left|\widehat{e}_{1}\right|\right)\left(1+\left|\widehat{e}_{1}\right|\right)\right)\right],
\end{array}
$$

where $\widehat{e}_{1}=z_{1}-\vartheta^{*}$ and $\widehat{e}_{2}=z_{2}-\dot{\vartheta}^{*}, z_{1}$ and $z_{2}$ are the state estimations from AESO, and $\widehat{F}_{1}$ is the estimation of the "total disturbance" from AESO. $\vartheta^{*}$ and $\dot{\vartheta}^{*}$ are the reference from command filter [32], and $\widehat{s}=\widehat{e}_{1}+(1 / \alpha) \widehat{e}_{1}{ }^{a / b}+(1 / \beta) \widehat{e}_{2}{ }^{p / q}+$ $(1 / \eta)\left|\widehat{e}_{1}\right| \exp \left(\left|\widehat{e}_{1}\right|\right)$.

Proof. Consider the following Lyapunov function:

$$
V=\frac{1}{2} s^{2} .
$$

Therefore,

$$
\begin{aligned}
\dot{V}=s \dot{s}= & s\left(e_{2}\left(1+\frac{a}{\alpha b} \widehat{e}_{1}^{a / b-1}+\frac{1}{\eta} \exp \left(\left|\widehat{e}_{1}\right|\right)\left(1+\left|\widehat{e}_{1}\right|\right)\right)\right. \\
& \left.+\frac{p}{\beta q} e_{2}^{p / q-1} \dot{e}_{2}\right) .
\end{aligned}
$$

Substituting the control (33) and system (2) into (35) yields

$$
\begin{aligned}
\dot{V}= & s\left(e_{2}\left(1+\frac{a}{\alpha b} \widehat{e}_{1}^{a / b-1}+\frac{1}{\eta} \exp \left(\left|\widehat{e}_{1}\right|\right)\left(1+\left|\widehat{e}_{1}\right|\right)\right)\right. \\
& \left.+\frac{p}{\beta q} e_{2}^{p / q-1}\left(F_{1}+b_{1} u-\ddot{\vartheta}^{*}\right)\right) \\
= & s\left(e_{2}\left(1+\frac{a}{\alpha b} \widehat{e}_{1}^{a / b-1}+\frac{1}{\eta} \exp \left(\left|\widehat{e}_{1}\right|\right)\left(1+\left|\widehat{e}_{1}\right|\right)\right)\right. \\
& +\frac{p}{\beta q} e_{2}^{p / q-1}\left(F_{1}-\beta \frac{q}{p}\left(\phi \widehat{s}+\gamma \widehat{s}^{m / n}\right)-\beta \frac{q}{p} \widehat{e}_{2}{ }^{2-p / q}\right. \\
& \left.\left.\cdot\left(1+\frac{a}{\alpha b} \widehat{e}_{1}{ }^{a / b-1}+\frac{1}{\eta} \exp \left(\left|\widehat{e}_{1}\right|\right)\left(1+\left|\widehat{e}_{1}\right|\right)\right)-\left(\widehat{F}_{1}-\ddot{\vartheta}^{*}\right)-\ddot{\vartheta}^{*}\right)\right) \\
= & s\left(e_{2}\left(1+\frac{a}{\alpha b} \widehat{e}_{1}^{a / b-1}+\frac{1}{\eta} \exp \left(\left|\widehat{e}_{1}\right|\right)\left(1+\left|\widehat{e}_{1}\right|\right)\right)\right. \\
& +\frac{p}{\beta q} e_{2}^{p / q-1}\left(F_{1}-\widehat{F}_{1}-\beta \frac{q}{p}\left(\phi \widehat{s}+\gamma \widehat{s}^{m / n}\right)-\beta \frac{q}{p} \widehat{e}_{2}{ }^{2-p / q}\right. \\
& \left.\left.+\left(1+\frac{a}{\alpha b} \widehat{e}_{1}{ }^{a / b-1}+\frac{1}{\eta} \exp \left(\left|\widehat{e}_{1}\right|\right)\left(1+\left|\widehat{e}_{1}\right|\right)\right)\right)\right) .
\end{aligned}
$$

Then,

$$
\begin{aligned}
\dot{V}= & s\left(e_{2}\left(1+\frac{a}{\alpha b} \widehat{e}_{1}^{a / b-1}+\frac{1}{\eta} \exp \left(\left|\widehat{e}_{1}\right|\right)\left(1+\left|\widehat{e}_{1}\right|\right)\right)\right. \\
& +\frac{p}{\beta q} e_{2}^{p / q-1}\left(\tilde{F}_{1}-\beta \frac{q}{p}\left(\phi(s-\tilde{s})+\gamma(s-\tilde{s})^{m / n}\right)\right. \\
& \left.\left.-\beta \frac{q}{p} \widehat{e}_{2}^{2-p / q}\left(1+\frac{a}{\alpha b} \widehat{e}_{1}^{a / b-1}+\frac{1}{\eta} \exp \left(\left|\widehat{e}_{1}\right|\right)\left(1+\left|\widehat{e}_{1}\right|\right)\right)\right)\right) \\
= & l\left(e_{2}\right) s\left(\left(e_{2}^{2-p / q}-\widehat{e}_{2}^{2-p / q}\right)\right. \\
& +\frac{a}{\alpha b}\left(e_{2}^{2-p / q} e_{1}^{a / b-1}-\widehat{e}_{2}^{2-p / q} \widehat{e}_{1}^{a / b-1}\right) \\
& +\left(e_{2}^{2-p / q} \exp \left(\left|e_{1}\right|\right)\left(1+\left|e_{1}\right|\right)-\widehat{e}_{2}^{2-p / q} \exp \left(\left|\widehat{e}_{1}\right|\right)\left(1+\left|\widehat{e}_{1}\right|\right)\right) \\
& \left.+\frac{\beta q}{p} \tilde{F}_{1}+\left(\phi \tilde{s}+\gamma \tilde{s}^{m / n}\right)-\left(\phi s+\gamma s^{m / n}\right)\right),
\end{aligned}
$$

where $\tilde{F}_{1}=F_{1}-\widehat{F}_{1}$ and $\tilde{s}=s-\widehat{s}$. Note that $\tilde{e}_{1}=e_{1}-\widehat{e}_{1}$ and $\tilde{e}_{2}=e_{2}-\widehat{e}_{2}$, then according to the inequation theorem:

$$
\begin{aligned}
& e_{2}^{2-p / q}-\widehat{e}_{2}{ }^{2-p / q} \leq\left|\tilde{e}_{2}\right|^{2-p / q}, \\
& e_{2}^{2-p / q} e_{1}{ }^{a / b-1}-\widehat{e}_{2}^{2-p / q} \widehat{e}_{1}{ }^{a / b-1} \\
& =e_{2}{ }^{2-p / q} e_{1}{ }^{a / b-1}-\widehat{e}_{2}{ }^{2-p / q}\left(e_{1}-\tilde{e}_{1}\right)^{a / b-1} \\
& \leq e_{1}^{a / b-1} e_{2}^{2-p / q}-e_{1}^{a / b-1}{\widehat{e}_{2}}^{2-p / q}+\tilde{e}_{1}{ }^{a / b-1} \widehat{e}_{2}{ }^{2-p / q} \\
& =e_{1}^{a / b-1}\left(e_{2}^{2-p / q}-\widehat{e}_{2}^{2-p / q}\right)+\tilde{e}_{1}^{a / b-1} \widehat{e}_{2}^{2-p / q} \\
& \leq e_{1}^{a / b-1}\left|\tilde{e}_{2}\right|^{2-p / q}+\widehat{e}_{2}{ }^{2-p / q} \tilde{e}_{1}{ }^{a / b-1}, \\
& e_{2}^{2-p / q} \exp \left(\left|e_{1}\right|\right)\left(1+\left|e_{1}\right|\right)-\widehat{e}_{2}^{2-p / q} \exp \left(\left|\widehat{e}_{1}\right|\right)\left(1+\left|\widehat{e}_{1}\right|\right) \\
& =\exp \left(\left|e_{1}\right|\right)\left(1+\left|e_{1}\right|\right) e_{2}^{2-p / q}-\exp \left(\left|\hat{e}_{1}\right|\right)\left(1+\left|\hat{e}_{1}\right|\right) \\
& \cdot\left(e_{2}-\tilde{e}_{2}\right)^{2-p / q} \\
& \leq e_{2}^{2-p / q}\left(\exp \left(\left|e_{1}\right|\right)\left(1+\left|e_{1}\right|\right)-\exp \left(\left|\widehat{e}_{1}\right|\right)\left(1+\left|\widehat{e}_{1}\right|\right)\right) \\
& +\exp \left(\left|\widehat{e}_{1}\right|\right)\left(1+\left|\hat{e}_{1}\right|\right) \tilde{e}_{2}^{2-p / q} \\
& =e_{2}{ }^{2-p / q}\left(\exp \left(\left|\widehat{e}_{1}+\tilde{e}_{1}\right|\right)\left(1+\left|\widehat{e}_{1}+\tilde{e}_{1}\right|\right)-\exp \left(\left|\widehat{e}_{1}\right|\right)\left(1+\left|\widehat{e}_{1}\right|\right)\right) \\
& +\exp \left(\left|\hat{e}_{1}\right|\right)\left(1+\left|\hat{e}_{1}\right|\right) \tilde{e}_{2}^{2-p / q} \\
& =e_{2}^{2-p / q} \exp \left(\left|\widehat{e}_{1}\right|\right)\left(\left(1+\left|e_{1}\right|\right) \exp \left(\left|\tilde{e}_{1}\right|\right)-\left(1+\left|\widehat{e}_{1}\right|\right)\right) \\
& +\exp \left(\left|\widehat{e}_{1}\right|\right)\left(1+\left|\widehat{e}_{1}\right|\right) \tilde{e}_{2}^{2-p / q} \text {. }
\end{aligned}
$$

Consequently,

$$
\begin{aligned}
\dot{V} \leq & l\left(e_{2}\right) s\left(\left|\tilde{e}_{2}\right|^{2-p / q}+\left(e_{1}^{a / b-1}\left|\tilde{e}_{2}\right|^{2-p / q}+\widehat{e}_{2}{ }^{2-p / q} \tilde{e}_{1}{ }^{a / b-1}\right)\right. \\
& +\left(e _ { 2 } ^ { 2 - p / q } \operatorname { e x p } ( | \widehat { e } _ { 1 } | ) \left(\left(1+\left|e_{1}\right|\right) \exp \left(\left|\tilde{e}_{1}\right|\right)\right.\right. \\
& \left.\left.-\left(1+\left|\widehat{e}_{1}\right|\right)\right)+\exp \left(\left|\widehat{e}_{1}\right|\right)\left(1+\left|\widehat{e}_{1}\right|\right) \tilde{e}_{2}^{2-p / q}\right) \\
& \left.+\left(\phi \tilde{s}+\gamma \tilde{s}^{m / n}\right)+\frac{p}{\beta q} \tilde{F}_{1}-\left(\phi s+\gamma s^{m / n}\right)\right) .
\end{aligned}
$$

Indeed, the errors $\tilde{e}_{1}, \tilde{e}_{2}$, and $\tilde{F}_{1}$ are the estimation errors by the AESO. Moreover, from Lemma 1 in Section 3.1, 
TABle 1: Parameters of the hose-drogue assembly.

\begin{tabular}{lcc}
\hline Parameters & Nomenclatures & Values \\
\hline$\mu$ & Mass per unit length & $4.11 \mathrm{~kg} / \mathrm{m}$ \\
$d_{k}$ & Diameter of hose & $0.067 \mathrm{~m}$ \\
$c_{t, k}$ & Skin friction coefficient & 0.001 \\
$c_{n, k}$ & Hose drag coefficient & 0.28 \\
$m_{\text {drogue }}$ & Mass of drogue & $29.5 \mathrm{~kg}$ \\
$d_{\text {drogue }}$ & Diameter of drogue & $0.61 \mathrm{~m}$ \\
$c_{\text {drogue }}$ & Drogue drag coefficient & 0.831 \\
$N$ & Number of links & 24 \\
$H_{0}$ & Flight altitude & $7620 \mathrm{~m}$ \\
$V_{0}$ & Airspeed & $550 \mathrm{~km} / \mathrm{h}$ \\
\hline
\end{tabular}

it can be certainly known that these estimation errors $\left(\tilde{e}_{1}, \tilde{e}_{2}\right.$, and $\left.\tilde{F}_{1}\right)$ are uniformly bounded $\left|\tilde{e}_{1}\right|<\varepsilon_{e_{1}},\left|\tilde{e}_{2}\right|<\varepsilon_{e_{2}}$, and $\left|\tilde{F}_{1}\right|<\varepsilon_{F_{1}}$, and the bounds $\varepsilon_{e_{1}}, \varepsilon_{e_{2}}$, and $\varepsilon_{F_{1}}$ are deterministic if Assumption 1-3 are satisfied. Namely, although states and disturbance are estimated in the probabilistic framework by AESO, the bounds of the estimation errors are deterministic. Based on this conclusion, we continue the proof using the deterministic Lyapunov function.

Consequently, the estimation error $\tilde{F}_{1}$ can be small enough and uniformly bounded via properly turning the parameters of AESO according to Lemma 1 . As $0.5<p / q<1$ and $\beta$ is selected big enough generally, the $(p /(\beta q)) \tilde{F}_{1}$ will be small enough.

Besides, $\tilde{e}_{1}$ and $\tilde{e}_{2}$ are the estimation errors of AESO, and they also are uniformly bounded according to Lemma 1 . As $\tilde{s}$ is the bounded function of $\tilde{e}_{1}$ and $\tilde{e}_{2}$, it is also uniformly bounded. Assume $\Delta$ is the function of the residual error from AESO,

$$
\begin{aligned}
\Delta= & \left|\tilde{e}_{2}\right|^{2-p / q}+\left(e_{1}^{a / b-1}\left|\tilde{e}_{2}\right|^{2-p / q}+\widehat{e}_{2}^{2-p / q} \tilde{e}_{1}^{a / b-1}\right) \\
& +\left(e_{2}{ }^{2-p / q} \exp \left(\left|\widehat{e}_{1}\right|\right)\left(\left(1+\left|e_{1}\right|\right) \exp \left(\left|\tilde{e}_{1}\right|\right)-\left(1+\left|\widehat{e}_{1}\right|\right)\right)\right. \\
& \left.+\exp \left(\left|\widehat{e}_{1}\right|\right)\left(1+\left|\widehat{e}_{1}\right|\right) \tilde{e}_{2}{ }^{2-p / q}\right)+\left(\phi \tilde{s}+\gamma \tilde{s}^{m / n}\right) .
\end{aligned}
$$

Based on the analysis above, the residual error $\Delta$ can be bounded by properly turning the parameters of AESO. Therefore, (39) can be written as

$$
\dot{V} \leq l\left(e_{2}\right)\left(\left(\Delta+\frac{p}{\beta q} \tilde{F}_{1}\right) s-\left(\phi s^{2}+\gamma s^{m / n+1}\right)\right) .
$$

Hence, $\dot{V}<0$ can be satisfied if the $\phi$ and $\gamma$ are chosen great enough. Then, $\vartheta \rightarrow \vartheta^{*}$ and $\omega \rightarrow \dot{\vartheta}^{*}$ can be achieved by the proposed control scheme for PMSM tracking control to suppress the hose vibration during AAR coupling. And from the controller (33), it can be further known that no occurring of singularity is ensured during the whole process.
TABLE 2: Parameters of the PMSM.

\begin{tabular}{lcc}
\hline Parameters & Nomenclatures & Values \\
\hline$R$ & Stator resistance & $1.65 \Omega$ \\
$L_{\mathrm{s}}$ & Stator inductance & $0.0092 \mathrm{H}$ \\
$P$ & Pole pairs & 4 \\
$\psi_{\mathrm{f}}$ & Stator flux linkage & $0.175 \mathrm{~Wb}$ \\
$J$ & Moment of inertia & $0.001 \mathrm{~kg} \cdot \mathrm{m}^{2}$ \\
$B$ & Viscous friction coefficient & $4.831 e^{-5} \mathrm{~N} \cdot \mathrm{m} \cdot \mathrm{s}$ \\
$i$ & Reduction ratio & 10 \\
$r$ & Radius of reel & $0.06 \mathrm{~m}$ \\
\hline
\end{tabular}
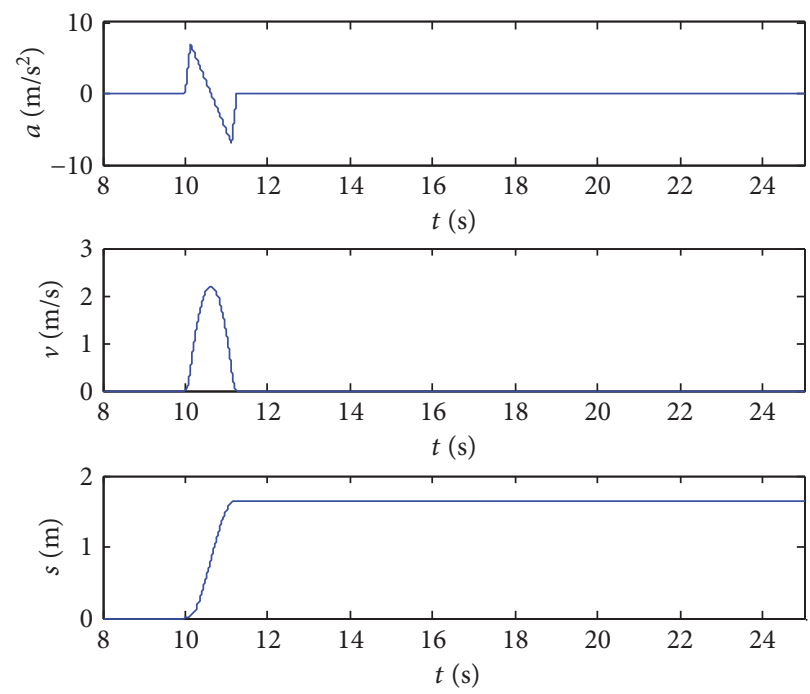

FIGURE 5: The relative coupling profile.

As described in Section 2, the current control loops are designed with proportion integral (PI) [18]. Here, two PI controllers are adopted in the two current loops to stabilize the current tracking errors of $d-q$ axes, respectively.

$$
\begin{aligned}
& u_{q}=k_{q p}\left(i_{q}{ }^{*}-i_{q}\right)+k_{q I} \int_{0}^{t}\left(i_{q}{ }^{*}-i_{q}\right) d t \\
& u_{d}=k_{d p}\left(i_{d}{ }^{*}-i_{d}\right)+k_{d I} \int_{0}^{t}\left(i_{d}{ }^{*}-i_{d}\right) d t .
\end{aligned}
$$

Generally, the reference $i_{d}{ }^{*}$ in the $\mathrm{d}$ current loop is set to be zero to maintain a constant flux operating condition, and the reference current $i_{q}{ }^{*}$ is determined by the subsystem $S_{1}$. Together with (33), the controller for PMSM in AAR is completed.

\section{Application to the Vibration Suppression of the Flexible Refueling Hose}

The vibration suppression problem of the refueling hose in AAR coupling is specially selected as an application example here for the proposed scheme due to its hostile working environment where all the assuming conditions presented in the 


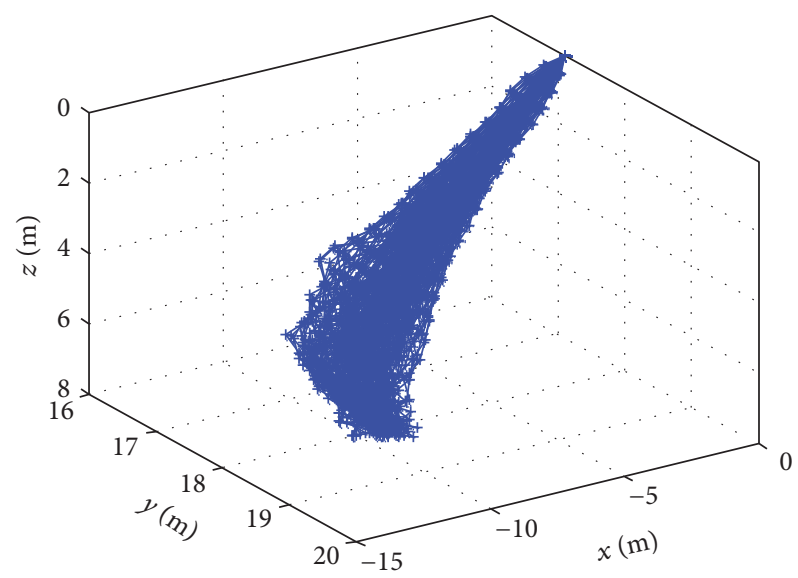

(a) Hose vibration history (10s-25s)-without vibration suppression

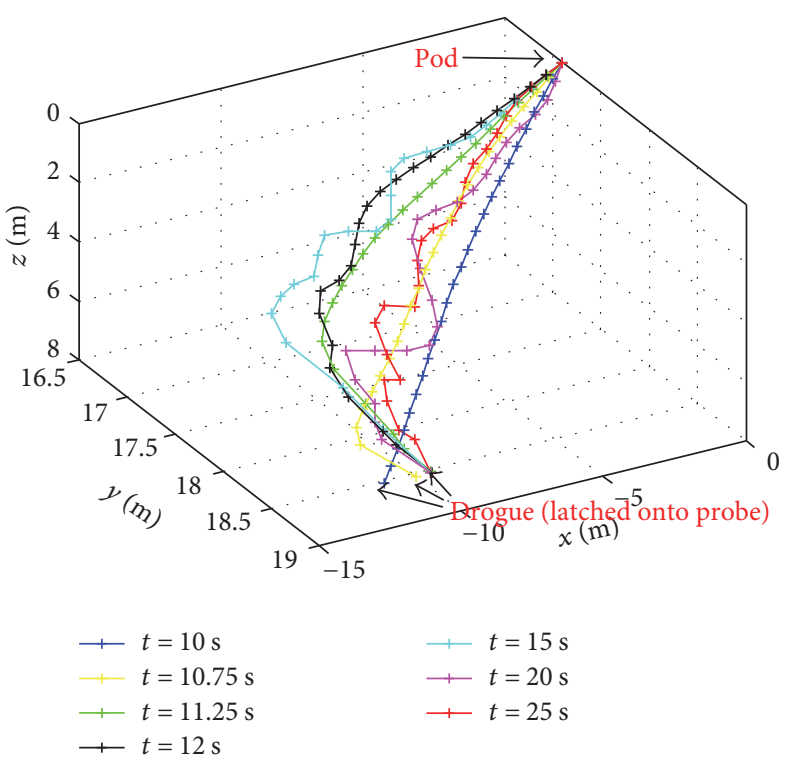

(b) Hose geometry history (selected time)—without vibration suppression

FIGURE 6: Flexible aerial refueling hose vibration during coupling without control scheme.

introduction section are satisfied. The features of the proposed method can be roundly verified in this application.

Compared with the traditional methods, in order to describe the complex dynamic characteristics of the host vibration more accurately, the hose-drogue assembly model with all links having variable length (by Wang et al.'s work [15] which is built based on the works of Ro et al. [14]) is adopted in this paper to model the hose-drogue. The parameters of the hose-drogue assembly and the flight condition are listed in Table 1. Table 2 lists the parameters of the PMSM used here.

Remark 6. For details of the model of the hose-drogue assembly (including the models for the skin friction, aerodynamic forces, and the kinematics and dynamics of the hosedrogue assembly under multiple flows in AAR), it can be found in [14] and [15]. Considering the primary motivation and purpose of this work, we will not repeat that in detail.

The initial hose length before the contact of the probe and drogue is set as $14.3 \mathrm{~m}$ here. And the longer distance the probe moves forward, the more violent the vibration goes. Therefore, in this paper, assume that the probe initially contacts the drogue at $10 \mathrm{~s}$ and then pushes the drogue forward along the relative high acceleration coupling profile [13], as illustrated in Figure 5.

The hose vibration without any suppression can be seen in Figure 6. Figure 6(a) shows the hose-drogue assembly vibration history from the time $t=10 \mathrm{~s}$ to $t=25 \mathrm{~s}$. The hose vibration envelope during the simulation time interval can be clearly seen in Figure 6(a). It can be seen that the hose vibrates at a high frequency, and the vibration amplitudes involve a wide space range. The refueling hose will vibrate more and more drastically with the time going on. Figure $6(\mathrm{~b})$ selects some specific time $(t=10,10.75,11.25$,
$12,15,20$, and $25 \mathrm{~s}$ ) to show the hose-drogue geometry history. It is obvious that the hose-drogue will shake execrably if no suppression scheme is introduced. It is a great threat to the safety of the AAR; the hose may even be broken off, and the receiver aircraft behind also may be damaged. The drastic shake of the hose indicates that there must be some control strategies to suppress the hose vibration for the safe AAR.

Figure 7 intuitively shows the shock forces on the hosedrogue assembly under vibration. As the drogue is pulled forward, the tension of the selected 24 joints (take the 1st, 13th, and 24th as examples) on the hose immediately decreases almost to zero owing to the instantaneous hose slackness. Then, the geometry of the hose changes, which will further increase the aerodynamic drags of the joints on the hose. As the aerodynamic drag choppily changes, the vibration goes more violent and travels toward the pod. As a result, the hose tension on the drogue and the force on the probe become more violent. The increasing forces will even break the PDR system and cause disastrous AAR accident. This drastic hose vibration caused by the excessive closure speed requires that there must be some vibration suppression strategies to ensure the flexible refueling hose-drogue assembly stable even under unexpected excessive closure speed and multiple disturbances.

The PMSM tracking control scheme above is applied to the vibration suppression of the refueling hose. To verify its effectiveness and superiority, the proposed NFTSM scheme with AESO and other two control schemes (linear ADRC $[6,33]$ with ESO and NTSM with AESO) are applied to the vibration suppression under strong disturbance and measurement noise. These methods are all designed based on the structure of cascade control loops (speed and current) shown in Section 2. The PI controller [21] is adopted for the current loop controller of these schemes. The 

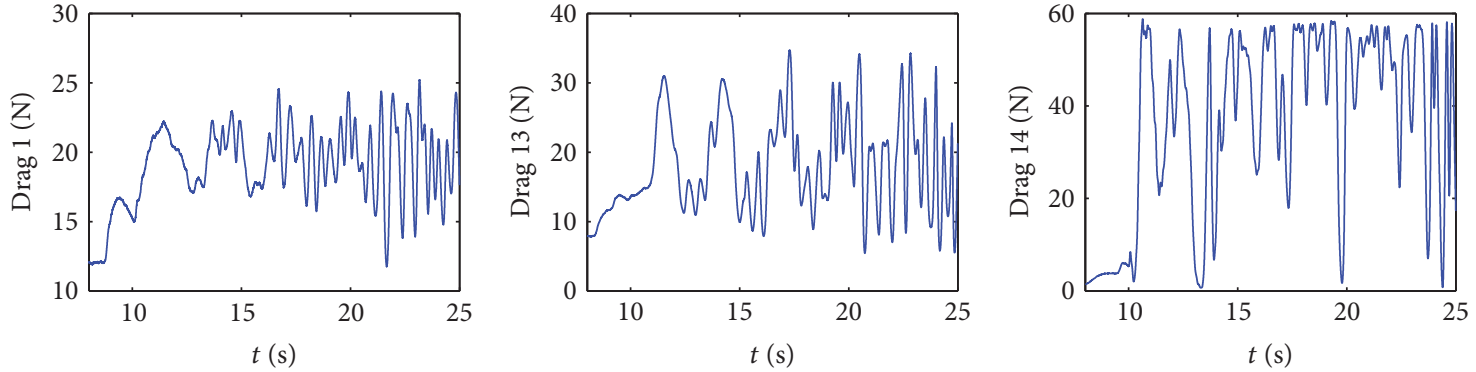

(a) Drag of the 1st, 13th, and 24th joint on the hose
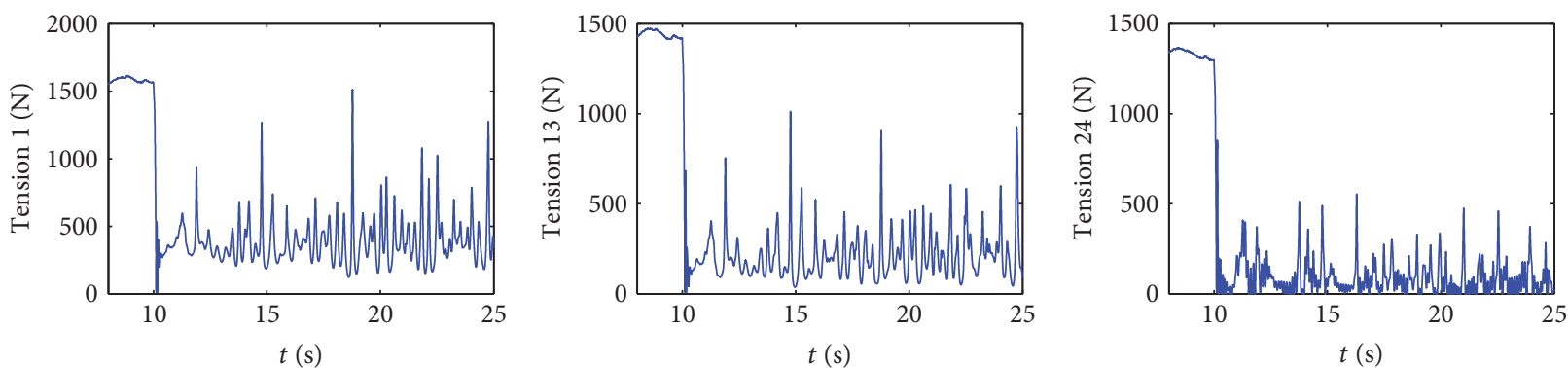

(b) Tension of the 1st, 13th, and 24th joint on the hose
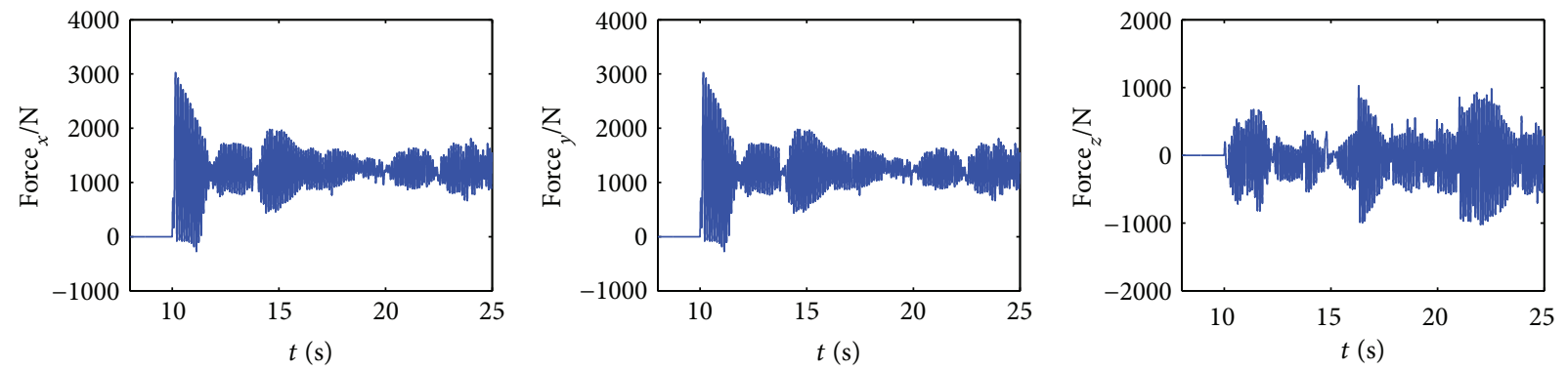

(c) Force on probe by the hose

FIGURE 7: Forces on hose and probe during coupling without vibration suppression.

parameters of the PI may vary from $10^{0}$ to $10^{3}$ for different PMSMs $[8,18]$. For comparison fairness, the PI parameters of these schemes $k_{p}, k_{I}$ are kept same, as shown in Table 3.

For system (2), a strong disturbance with multiple frequency characteristics is set as $d_{1}=3 e 3(\sin (\pi t)-0.5$ $\cos (5 \pi t)+0.5 \sin (10 \pi t))$. Besides, the Gaussian random noise with its variance $E\left(n_{i 1}{ }^{2}\right)=(1)^{2}$ is assumed to be the polluted noise superposed in the output of the system (2). And it is also assumed that the position of the probe in the vertical direction is disturbed by a sine disturbance as 0.01 $\sin (0.2 \pi t)$ after the coupling achieved due to the surrounding airflow disturbances, and the impact will finally appear in the PMSM system in the form of load disturbance and the fluctuation in the command signal. The other relevant parameters of these controllers are listed in Table 3. Contrastive simulations for these methods are conducted in MATLAB/SIMULINK 2011a and implemented on a personal computer with $3.2 \mathrm{GHz}$ Core i5 $\mathrm{CPU}$. The simulation sampling period is set as $0.001 \mathrm{~s}$.

It assumes that the control laws all come to work immediately here when the contact of the drogue and probe successfully achieved.
TABle 3: Parameters of the different control schemes.

\begin{tabular}{lcccc}
\hline $\begin{array}{l}\text { Control } \\
\text { schemes }\end{array}$ & Parameters & Values & Parameters & Values \\
\hline LADRC & $L_{\vartheta}$ & 200 & $K_{\vartheta}$ & {$[50,100]^{T}$} \\
with ESO & $k_{q p}, k_{d p}$ & 6 & $k_{q I}, k_{d I}$ & {$[600,600]^{T}$} \\
\hline NTSM & $p$ & 15 & $q$ & 13 \\
with & $\beta$ & 100 & $l_{1}$ & $5 \mathrm{e} 3$ \\
AESO & $\eta_{1}$ & $2 e 3$ & $k_{q p}, k_{q I}, k_{d p}, k_{d I}$ & {$[6,600,6,600]^{T}$} \\
\hline & $\bar{R}_{1}$ & $1^{2}$ & $\bar{Q}_{1}$ & $0.1^{2}$ \\
Proposed & $p$ & 15 & $q$ & 13 \\
NFTSM & $m$ & 17 & $b$ & 13 \\
with & $m$ & 11 & $n$ & 15 \\
AESO & $\alpha$ & 100 & $\beta$ & 100 \\
& $\phi$ & 100 & $\gamma$ & 100 \\
& $\eta$ & 100 & $k_{q p}, k_{q I}, k_{d p}, k_{d I}$ & {$[6,600,6,600]^{T}$} \\
\hline
\end{tabular}

$L_{\vartheta}$ is the bandwidth of the ESO in LADRC, and $K_{\vartheta}$ is the feedback controller gains of the PD in LADRC. 

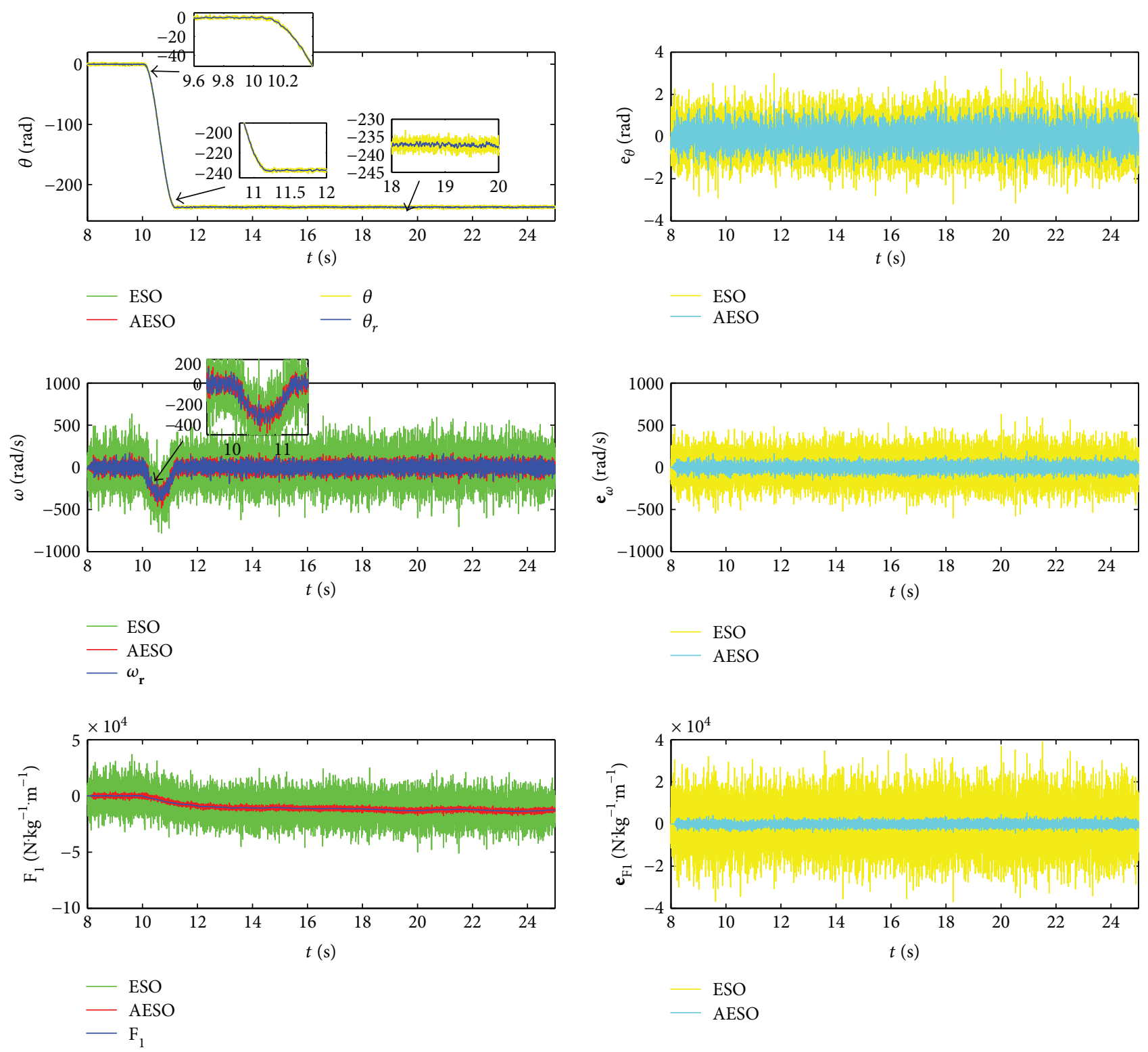

FIGURE 8: Estimation comparisons of ESO and AESO by the proposed NFTSM controller.

Firstly, to illustrate the superiority of AESO in reconstituting states and "total disturbance" of the system with measurement noise, the proposed NFTSM is used as the unified controller, and the comparison of ESO and AESO is conducted. Figure 7 shows the observation results of the two different observers.

In Figure $8, \vartheta_{r}$ and $\omega_{r}$ are the real values of the angular position and the angular velocity, respectively. $\vartheta$ is the measured value from the angular position sensor. Based on the same controller, it is obvious that AESO performs better than ESO when the sensor measurement noise exists. The reconstituted states and total disturbance of AESO are closer to the real values of the systems, and the observation errors are much smaller for all the states and disturbance. Although the existence of the measurement noise whose variance is $(1 \mathrm{rad})^{2}$, the angular tracking error $e_{\theta}$ is ensured within $2 \mathrm{ra}$ $\mathrm{d}$, which is much smaller than the estimation error by ESO.
A similar result can be found in the estimation angular velocity. The estimation errors for the total disturbance are especially obvious. It can be seen that the amplitude of the estimated disturbance $\widehat{F}_{1}$ by ESO may be ten times of the real value $F_{1}$, while the $\widehat{F}_{1}$ by AESO is much closer to the real value $F_{1}$ and the estimation error for $F_{1}$ by AESO is 5 to 10 times smaller than the ESO's. Therefore, AESO possesses the ability to well reconstitute the system states and disturbance even with measurement noise and strong disturbance.

Then, the time history of the hose geometries by different control schemes is shown in Figure 8, and the side view in $\mathrm{XOZ}$ plane for each scheme is also attached to the figures. It is clear that the vibration in LADRC is still serious on the condition that the disturbances and measurement noise are taken into consideration. However, the NTSM with AESO can suppress the vibration effectively in the same condition, 


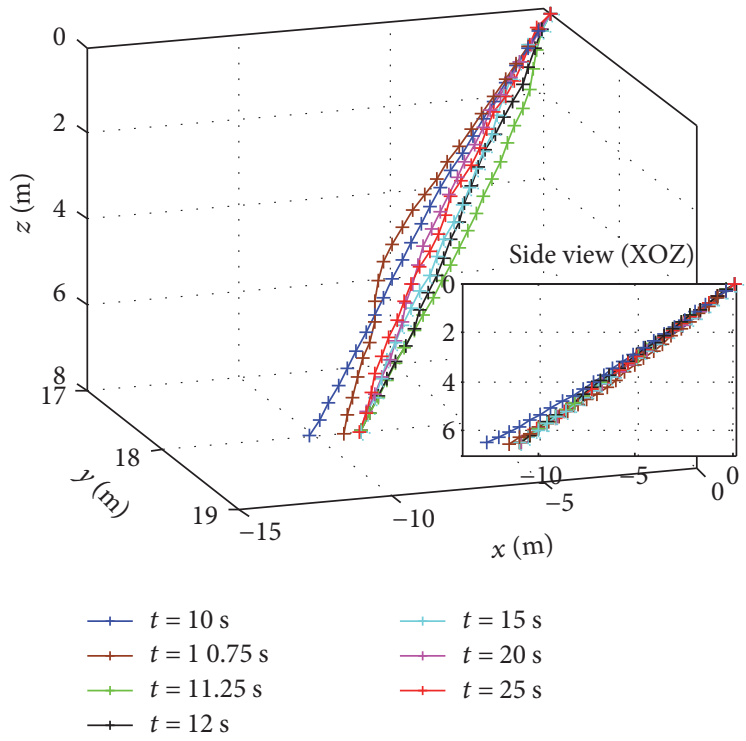

(a) Hose geometry history-LADRC

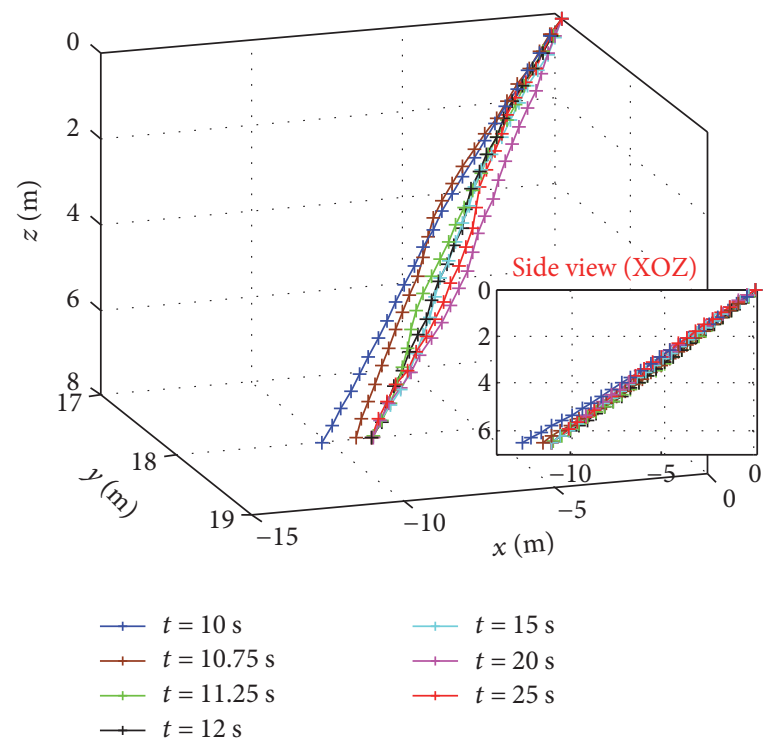

(b) Hose geometry history-NTSM

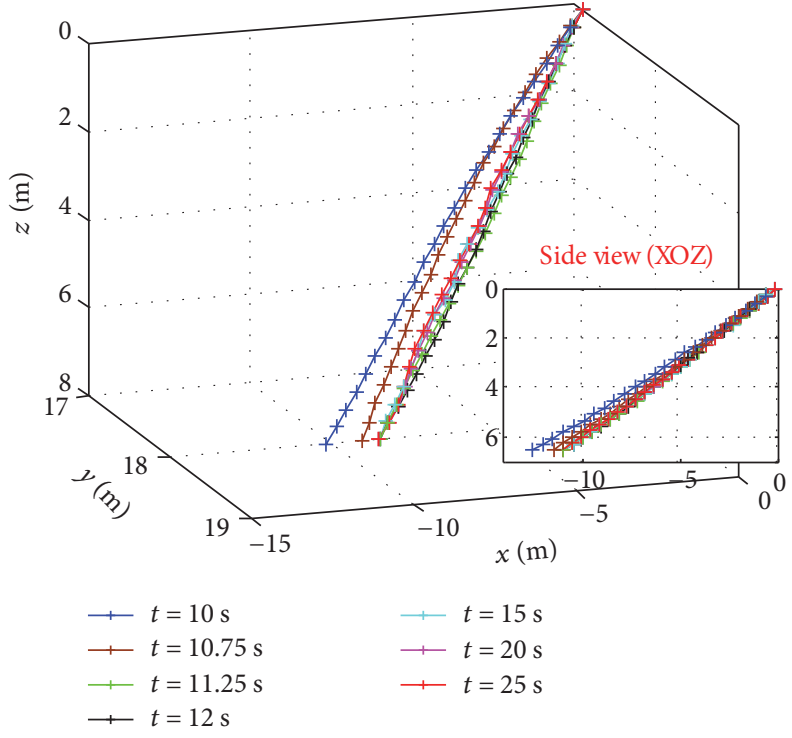

(c) Hose geometry history-NFTSM

Figure 9: The hose-drogue assembly's dynamic motions by different control scheme.

but its effectiveness is slightly poorer than the proposed NFTSM with AESO. From Figure 9, one can find that the hose geometries by the proposed NFTSM schemes look more stable and orderly. The proposed NFTSM with AESO cannot only track the command (transformed from the hose slack during the vibration) accurately in a shorter time. As the probe travels forward, the proposed controller can retract the hose rapidly and precisely and keep the catenary shape of the hose steady. The hose slack is maintained at the level just before coupling, and the hose vibration does not occur at all. With the compensation provided by the control laws, the catenary shape of the hose can always be kept steady. As a result, the vibration can be suppressed accurately and immediately. Figure 10 shows the drags, tensions on the 1 st, 13th, and 24th joint on the drogue, and the forces on probe caused by the vibration hose-drogue assembly.
Although the superiority of the proposed NFTSM scheme over the NTSM schemes has been verified via the hose geometry history in Figure 9, Figure 10 presents more visualized results to evidence that. From Figure 10, it can be seen that the proposed vibration scheme ensures the drag forces, tensions on the hose, and the forces on the probe all much smaller than those of the NTSM schemes. These smaller forces indicate that the hose-drogue assembly are more stable and organized under the proposed scheme. The vibration can be more effectively suppressed by the proposed NFTSM scheme.

From Figures 11, 12, 13, and 14, the states and relevant tracking errors of the PMSM subsystem are shown, respectively. The external load on the probe is shown in Figure 15. And the length histories of the hose reeling driven by the PMSM are shown in Figure 16. 

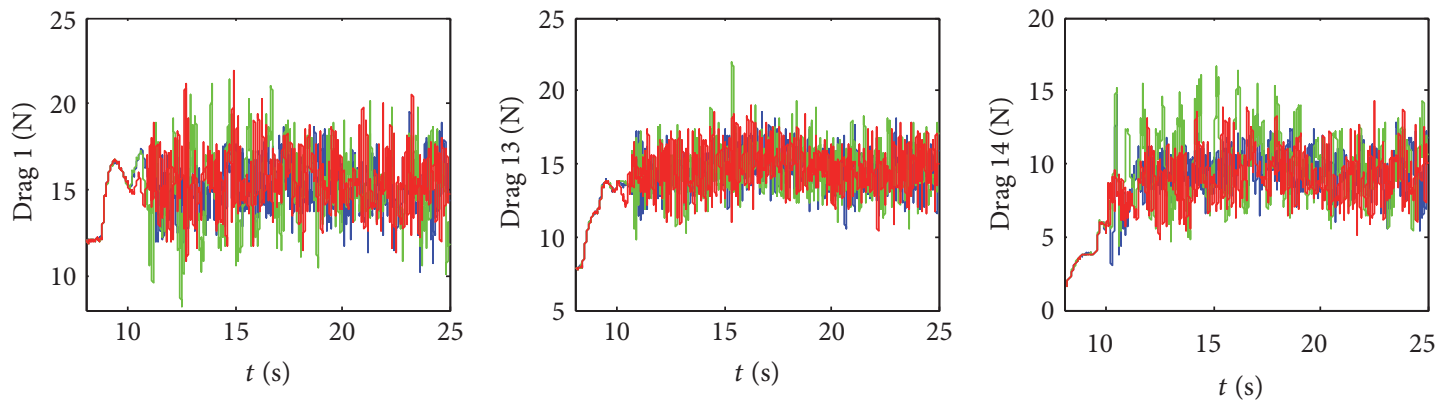

- LADRC
- NTSM
- NFTSM

(a) Drag of the 1st, 13th, and 24th joint on the hose
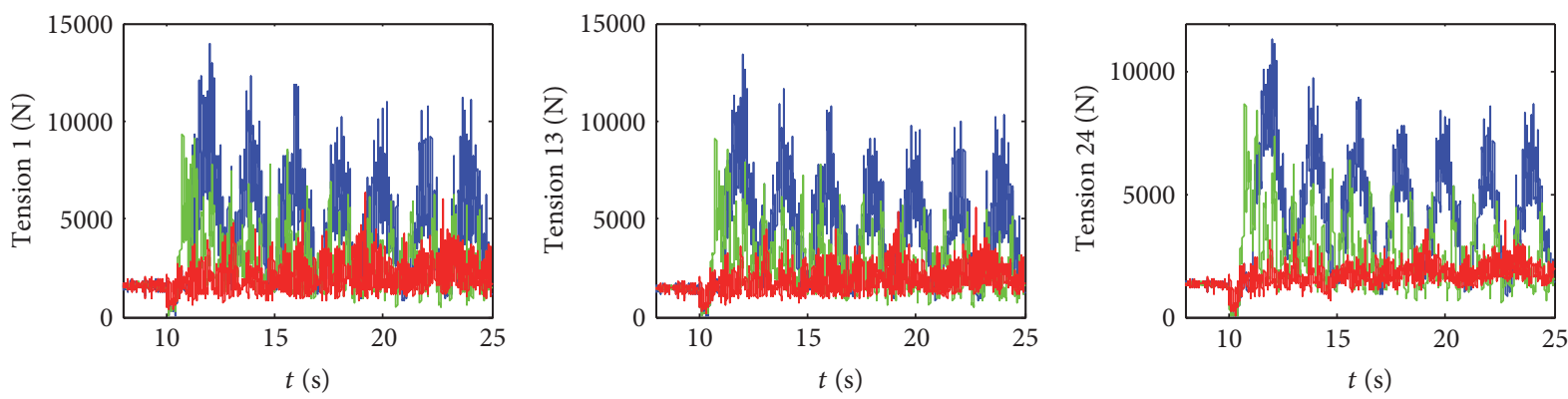

(b) Tension of the 1st, 13th, and 24th joint on the hose
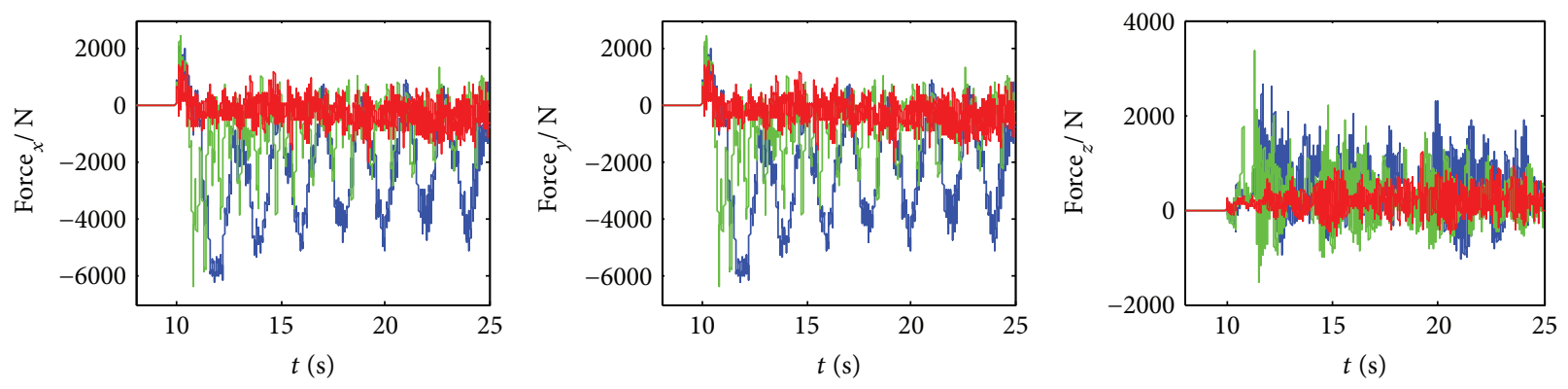

(c) Force on probe by the hose

FIgURE 10: Forces on hose and probe by different vibration suppression schemes.

From Figures 11 and 12, the influence of the sensor measurement noise to the system is clearly presented. It can also be inferred that when the external disturbance is big enough, it cannot be expected to track the command accurately by a single controller. The strong total disturbance, including the internal and external disturbance, should be observed by an observer and then compensated by the control laws. By the composition from AESO, the LADRC can track the command signal with strong disturbance and measurement noise. However, the simple PD controller in the LADRC limits the tracking accuracy and rapidity. The NTSM with AESO shows better track performance and robustness than the LADRC. However, by obvious observation of the tracking curve in Figure 11 and the tracking error in Figure 12, a distinct overshoot still exists nearby $t=10 \mathrm{~s}$. Namely, the mechanism of the NTSM still should be improved for better tracking performance especially the rapidity, because its global convergence is limited. By introducing the tracking

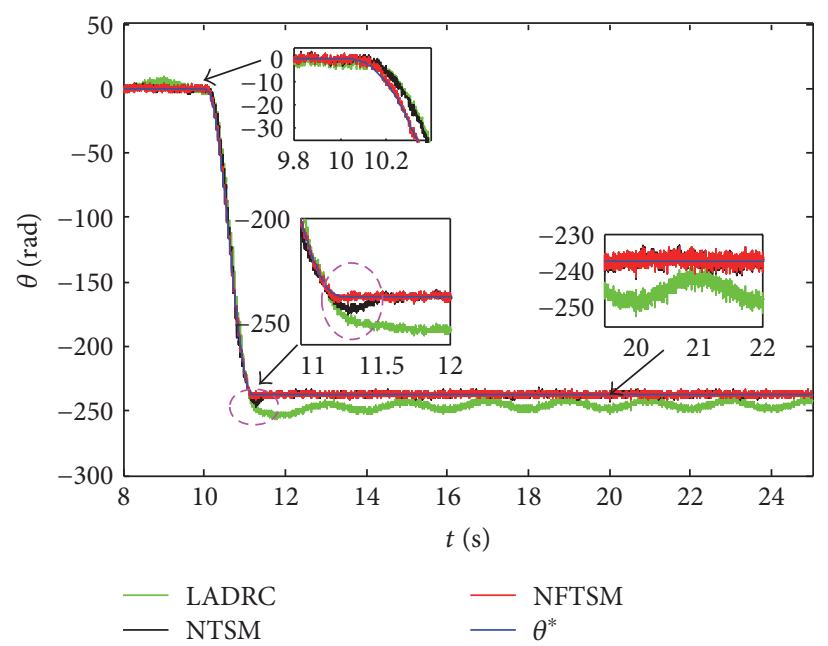

Figure 11: Angular position tracking results by different schemes. 


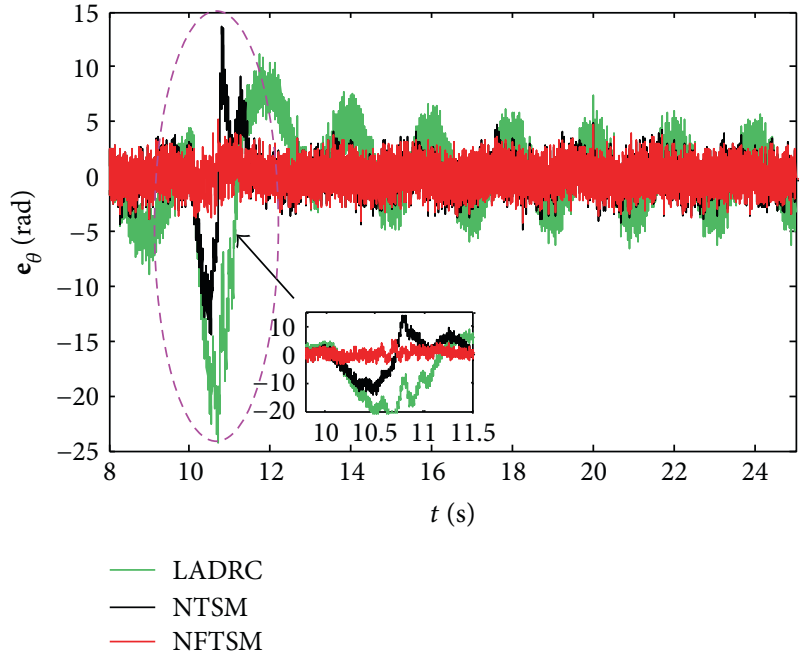

Figure 12: Angular position tracking errors by different schemes.

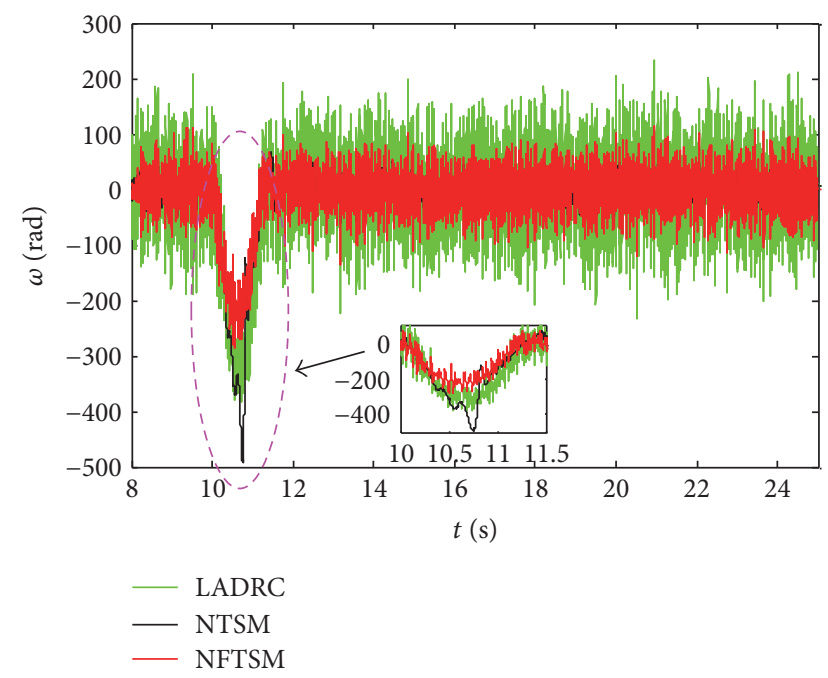

FIGURE 13: Angular velocity tracking results by different schemes.

error exponential term in sliding surface and specially designed reaching law, the proposed control scheme can accurately track the angular position command given by the hose in AAR with almost zero overshoot. Moreover, the angular tracking error is well guaranteed within 5 rad even under the existence of the angular sensor measurement noise with its variance $1^{2}$ rad and the strong total disturbance, as shown in Figure 11. And the angular velocity tracking error can also be ensured much smaller than that of LADRC. From the tracking result in Figures 11-14, it can be found that the proposed NFTSM with AESO shows the best performance in rapidity and precision than the other two.

From Figure 15, the external load by the LADRC is greater than the other two, and the periodic fluctuation is caused by the poorer tracking performance of the PD controller in LADRC. The external load by NTSM with AESO is about 0.5 times of the LADRC's. However, with the relatively poor transient characteristics (slower tracking speed

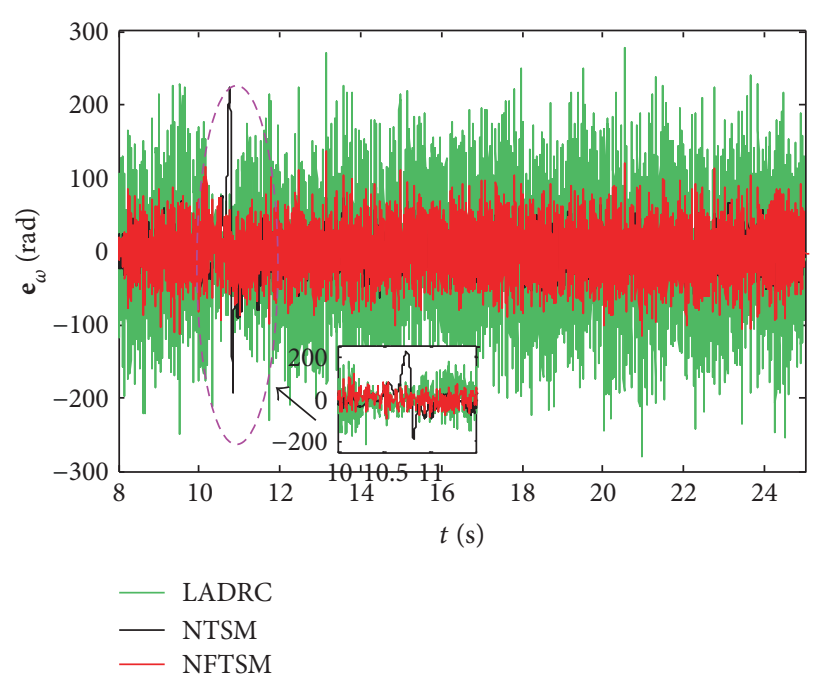

FIGURE 14: Angular velocity tracking errors by different schemes.

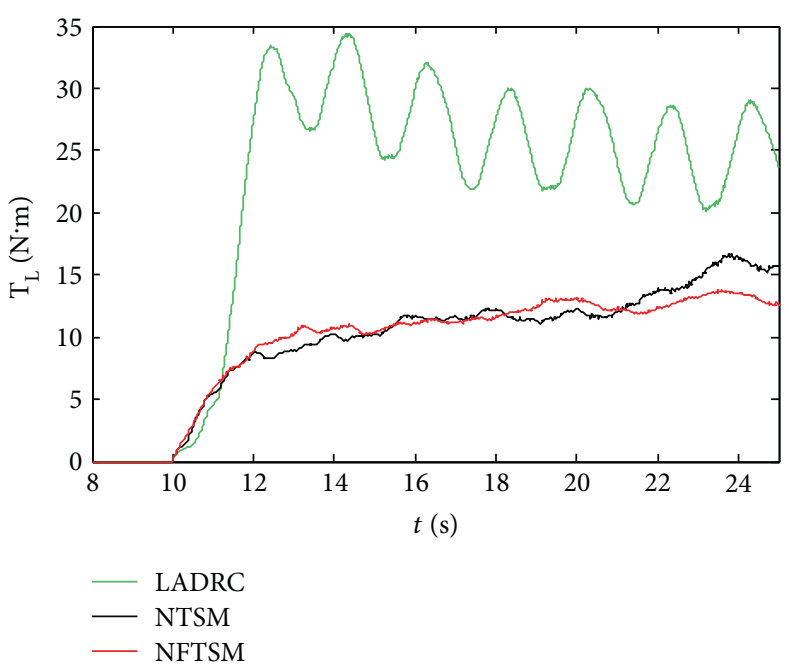

Figure 15: Load torques by different schemes.

and greater overshoot), the NTSM does not track the command rapidly enough compared to the proposed NFTSM. This relatively poor performance during the coupling process will lead to greater fluctuation of the external load as time goes on, which will finally affect the stability of the hosedrogue. It can be seen that the external load by NFTSM is no more than $15 \mathrm{~N} \cdot \mathrm{m}$ in Figure 15 throughout the refueling contact process. And although the external load by NTSM is very close to NFTSM's, the curve of $T_{\mathrm{L}}$ is the smoothest, which indicates that the dynamic of the hose-drogue by this method is the most stable one. Indeed, this kind of tracking performance is expected, because we always expect the hose-drogue to keep stable even with the perturbed contact process of probe and drogue in AAR. It should especially be addressed that there is little difference between 21 and $25 \mathrm{sec}$ onds for the load torques by the NTSM and NFTSM methods. From Figures 9 and 10, we know that the hose vibration by the NTSM method is still more drastic than 


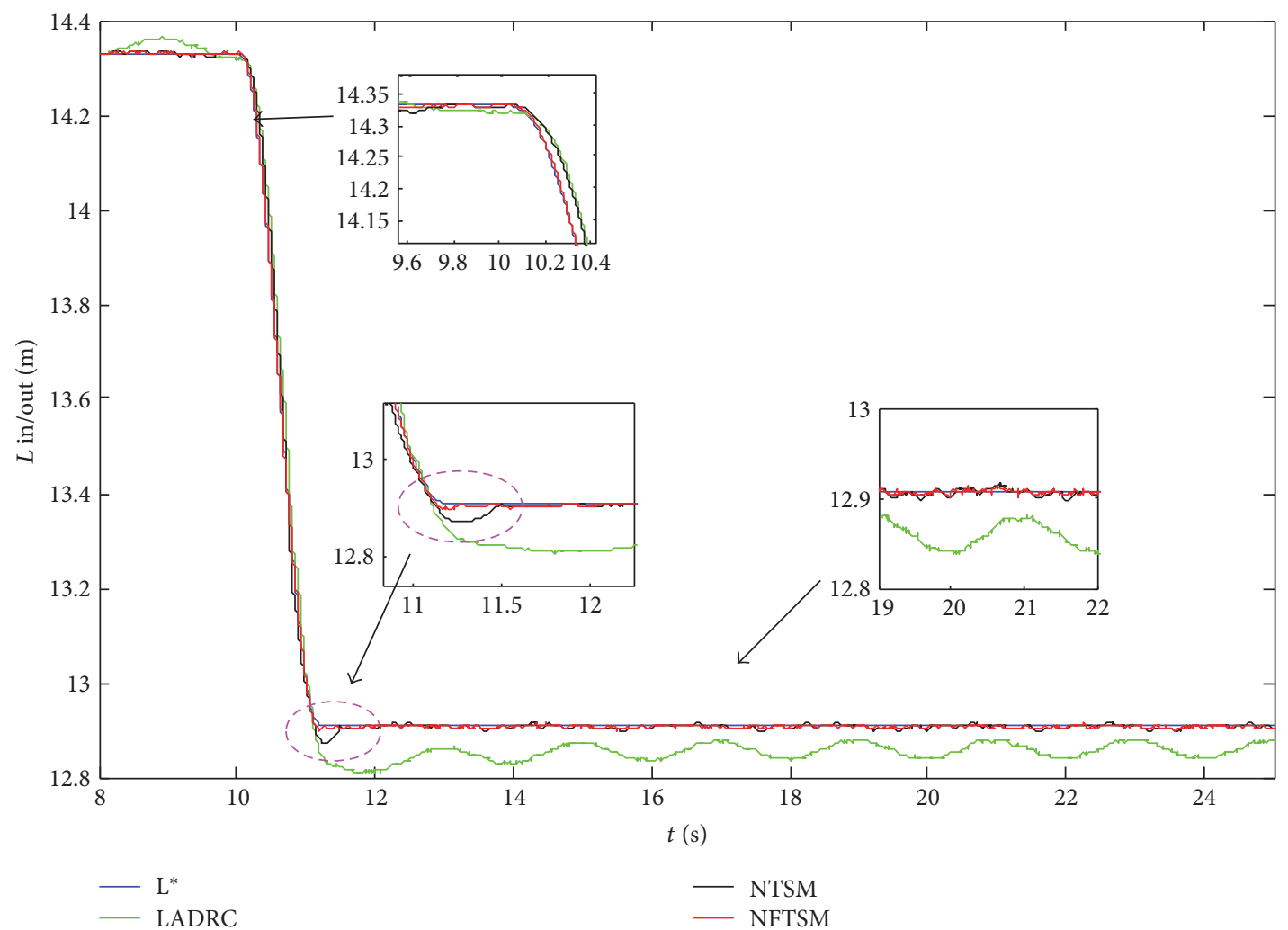

Figure 16: The hose length reeling in/out results by different schemes.

the NFTSM's. As time goes on, the vibration spreads along the flexible refueling hose under the impact of the unpredictable aerodynamic force behind the tanker aircraft. Then, according to the formulation $T_{\mathrm{L}}=(r / i)(1 /(s+1)) T_{1}$ in Section 2.2, the load torque $T_{\mathrm{L}}$ of the NTSM methods will change greatly and may even become larger than the load torque by the NFTSM method with the hose vibration method goes on.

From Figure 16, the length histories of the hose reeling by LADRC wave intensely due to the strong disturbance. That means that the degree of hose slack induced by coupling is still high, and the slackness of the hose is still severe. The refueling hose vibration may still go violent under the multiple disturbance and measurement noise, and the tension at the end of the hose will change drastically and rapidly. Similar to the angular position in Figure 11, an obvious overshoot also exists in the length of the hose by NTSM, and this overshoot will last about 0.5 seconds, while it can accurately track the angular position command which is given by the hose in AAR with almost zero overshoot. The hose can be precisely reeled in/out by the PMSM with almost no error and lag. The tracking performance of the proposed NFTSM with AESO is the best. And after the coupling, as the relative distance between the receiver and the tanker does not change anymore, the hose length is also maintained to be a constant. And the aerodynamic drags and tensions of the hose gradually become stable as well, then the vibration does not occur anymore.

\section{Conclusion}

The permanent magnet synchronous motor- (PMSM-) based take-up system is adopted for the aerial refueling hose vibration suppression in autonomous aerial refueling (AAR). A nonsingular fast terminal sliding-mode (NFTSM) control scheme with adaptive extended state observer (AESO) is designed for PMSM take-up system considering external disturbances and measurement noise. A sliding variable with tracking error exponential term is proposed and a special reaching law is designed to enhance the global convergence speed as well as the precision of the controller. The AESO is used to reconstitute the states and "total disturbance" of PMSM with the uncertainties both in the plant and the sensors. Then, the proposed control scheme for PMSM is applied to the flexible refueling hose vibration suppression during AAR coupling. Simulations demonstrate that the proposed scheme is more competitive in global convergence speed and accuracy for vibration suppression with PMSM.

\section{Appendix}

\section{The Derivational Process of (14)}

When the system errors $\left(e_{1}, e_{2}\right)$ reach the sliding surface $s=0$ and with (13), we get

$$
s=e_{1}+\frac{1}{\beta} e_{2}^{p / q}=0 .
$$


$e_{2}=\dot{e}_{1}=\dot{\vartheta}-\dot{\vartheta}^{*}=\omega-\dot{\vartheta}^{*} ;$ that is, $e_{2}^{p / q}=\dot{e}_{1}^{p / q}=-\beta e_{1}$. Define $V_{n}=0.5 e_{1}^{2}$; it can be got that $e_{1}^{2}=2 V_{n}$ and $\dot{V}_{n}=$ $e_{1} \dot{e}_{1}$ and then

$$
\dot{V}_{n}^{p / q}=e_{1}^{p / q} \dot{e}_{1}^{p / q}=-\beta e_{1}^{p / q} e_{1}=-\beta e_{1}^{(p+q) / q} .
$$

As $p$ and $q$ are all positive odd numbers, hence, $V_{n}<0$ is satisfied when $e_{1} \neq 0$. Based on the Lyapunov stability criterion, the dynamic $s=e_{1}+(1 / \beta) e_{2}{ }^{p / q}=0$ is stable, and the system states will converge to the equilibrium. And from $\dot{e}_{1}^{p / q}=-\beta e_{1}$ (A.2), it can be known that the only equilibrium is $e_{1}=0$. To get the convergence time $T_{n}$, we submit $e_{1}^{2}=2 V_{n}$ into (A.2) and get

$$
\dot{V}_{n}^{p / q}=-2^{(p+q) / 2 q} \beta V_{n}^{(p+q) / 2 q} .
$$

As $\dot{V}_{n}=\left(d V_{n}\right) /(d t)$, the following is obtained:

$$
\begin{aligned}
d t & =\frac{d V_{n}}{\dot{V}_{n}}=\frac{d V_{n}}{-\left(-\dot{V}_{n}\right)}=-\left(\left(-\dot{V}_{n}\right)^{p / q}\right)^{-q / p} d V_{n} \\
& =-\left(2^{(p+q) / 2 q} \beta V_{n}^{(p+q) / 2 q}\right)^{-q / p} d V_{n} .
\end{aligned}
$$

Furthermore, as $V_{n}\left(T_{n}\right)=0$ and $V_{n}(0)=0.5 e_{1}^{2}(0) \geq 0$, we integrate (A.4) from both sides.

$$
T_{n}=\int_{0}^{V_{n}(0)} 2^{(p+q) / 2 q} \beta V_{n}^{(p+q) / 2 q} d V_{n}
$$

\section{Conflicts of Interest}

The authors declare that they do not have any conflicts of interest to this work.

\section{Acknowledgments}

This work is supported by the National Natural Science Foundations of China under Grants 61673042 and 61473012, and Program for Changjiang Scholars and Innovative Research Team in University (Grant no. IRT13004). The authors especially like to thank Dr. Haitao Wang for his suggestions on the first version.

\section{References}

[1] G. Gramajo and P. Shankar, "An efficient energy constraint based UAV path planning for search and coverage," International Journal of Aerospace Engineering, vol. 2017, Article ID 8085623, 13 pages, 2017.

[2] Y. Zhu, X. Chen, and C. Li, "A moving frame trajectory tracking method of a flying-wing UAV using the differential geometry," International Journal of Aerospace Engineering, vol. 2016, Article ID 3406256, 9 pages, 2016.

[3] P. R. Thomas, U. Bhandari, S. Bullock, T. S. Richardson, and J. L. du Bois, "Advances in air to air refuelling," Progress in Aerospace Sciences, vol. 71, pp. 14-35, 2014.

[4] Z. Liu, J. Liu, and W. He, "Modeling and vibration control of a flexible aerial refueling hose with variable lengths and input constraint," Automatica, vol. 77, pp. 302-310, 2017.
[5] Z. Su, H. Wang, X. Shao, and Y. Huang, "A robust backstepping based trajectory tracking controller for the tanker with strict posture constraints under unknown flow perturbations," Aerospace Science and Technology, vol. 56, pp. 34-45, 2016.

[6] Z. Su, H. Wang, P. Yao, Y. Huang, and Y. Qin, "Back-stepping based anti-disturbance flight controller with preview methodology for autonomous aerial refueling," Aerospace Science and Technology, vol. 61, pp. 95-108, 2017.

[7] Z. Su, H. Wang, N. Li, Y. Yu, and J. Wu, "Exact docking flight controller for autonomous aerial refueling with back-stepping based high order sliding mode," Mechanical Systems and Signal Processing, vol. 101, pp. 338-360, 2018.

[8] J. L. Hansen, J. E. Murray, and N. V. Campos, "The NASA Dryden AAR project: a flight test approach to an aerial refueling system," in AIAA Atmospheric Flight Mechanics Conference and Exhibit, pp. 2004-4939, Providence, RI, USA, August 2004, AIAA.

[9] H. Duan and Q. Zhang, "Visual measurement in simulation environment for vision-based UAV autonomous aerial refueling," IEEE Transactions on Instrumentation and Measurement, vol. 64, no. 9, pp. 2468-2480, 2015.

[10] K. Ro and J. W. Kamman, "Modeling and simulation of hoseparadrogue aerial refueling systems," Journal of Guidance, Control, and Dynamics, vol. 33, no. 1, pp. 53-63, 2010.

[11] Z. Liu, J. Liu, and W. He, "Vibration control of a flexible aerial refuelling hose with input saturation," International Journal of Systems Science, vol. 48, no. 5, pp. 971-983, 2016.

[12] Z. H. Zhu and S. A. Meguid, "Elastodynamic analysis of aerial refueling hose using curved beam element," AIAA Journal, vol. 44, no. 6, pp. 1317-1324, 2006.

[13] J. C. Vassberg, D. T. Yeh, A. J. Blair, and J. M. Evert, "Numerical simulations of KC-10 wing-mount aerial refueling hosedrogue dynamics with a reel take-up system," in 21st AIAA Applied Aerodynamics Conference, pp. 2003-3508, Orlando, FL, USA, June 2003, AIAA.

[14] K. Ro, T. Kuk, and J. W. Kamman, "Dynamics and control of hose-drogue refueling systems during coupling," Journal of Guidance, Control, and Dynamics, vol. 34, no. 6, pp. 16941708, 2011.

[15] H. Wang, X. Dong, J. Xue, and J. Liu, "Dynamic modeling of a hose-drogue aerial refueling system and integral sliding mode backstepping control for the hose whipping phenomenon," Chinese Journal of Aeronautics, vol. 27, no. 4, pp. 930-946, 2014.

[16] R. E. Alden, G. G. Vennero, and Whittaker Controls Inc., assignee, "Aerial refueling system," US Patent 5141178, 1992.

[17] J. Linares-Flores, C. García-Rodríguez, H. Sira-Ramirez, and O. D. Ramirez-Cardenas, "Robust backstepping tracking controller for low-speed PMSM positioning system: design, analysis, and implementation," IEEE Transactions on Industrial Informatics, vol. 11, no. 5, pp. 1130-1141, 2015.

[18] S. Li, H. Liu, and S. Ding, "A speed control for a PMSM using finite-time feedback control and disturbance compensation," Transactions of the Institute of Measurement and Control, vol. 32, no. 2, pp. 170-187, 2010.

[19] J. Yang, W.-H. Chen, S. Li, L. Guo, and Y. Yan, "Disturbance/ uncertainty estimation and attenuation techniques in PMSM drives-a survey," IEEE Transactions on Industrial Electronics, vol. 64, no. 4, pp. 3273-3285, 2017. 
[20] D.-H. Ha, C.-S. Lim, and D.-S. Hyun, "Robust optimal nonlinear control with observer for position tracking of permanent magnet synchronous motors," Journal of Power Electronics, vol. 13, no. 6, pp. 975-984, 2013.

[21] S. Zheng, X. Tang, B. Song, S. Lu, and B. Ye, "Stable adaptive PI control for permanent magnet synchronous motor drive based on improved JITL technique," ISA Transactions, vol. 52, no. 4, pp. 539-549, 2013.

[22] Y. A.-R. I. Mohamed and E. F. el-Saadany, "A current control scheme with an adaptive internal model for torque ripple minimization and robust current regulation in PMSM drive systems," IEEE Transactions on Energy Conversion, vol. 23, no. 1, pp. 92-100, 2008.

[23] Y. Shtessel, C. Edwards, L. Fridman, and A. Levant, Sliding Mode Control and Observation, Springer, New York, 2014.

[24] H. Wang, W. Zhang, T. Yang, and Q. Qu, "Sliding mode control for diesel engine using extended state observer," Studies in Informatics \& Control, vol. 24, no. 4, pp. 439-448, 2015.

[25] M. Bahrami, B. Ebrahimi, and G. R. Ansarifar, "Sliding mode observer and control design with adaptive parameter estimation for a supersonic flight vehicle," International Journal of Aerospace Engineering, vol. 2010, Article ID 474537, 9 pages, 2010.

[26] H. P. Wang, D. Zheng, and Y. Tian, "High pressure common rail injection system modeling and control," ISA Transactions, vol. 63, pp. 265-273, 2016.

[27] L. Yang and J. Yang, "Nonsingular fast terminal sliding-mode control for nonlinear dynamical systems," International Journal of Robust and Nonlinear Control, vol. 21, no. 16, pp. 1865-1879, 2011.

[28] M. Chen, Q.-X. Wu, and R.-X. Cui, “Terminal sliding mode tracking control for a class of SISO uncertain nonlinear systems," ISA Transactions, vol. 52, no. 2, pp. 198-206, 2013.

[29] B. Zhao and J. Zhou, "Smooth adaptive finite time guidance law with impact angle constraints," International Journal of Aerospace Engineering, vol. 2016, Article ID 5730168, 19 pages, 2016.

[30] W. Xue, W. Bai, S. Yang, K. Song, Y. Huang, and H. Xie, "ADRC with adaptive extended state observer and its application to air-fuel ratio control in gasoline engines," IEEE Transactions on Industrial Electronics, vol. 62, no. 9, pp. 5847-5857, 2015.

[31] X. Zhang, L. Sun, K. Zhao, and L. Sun, "Nonlinear speed control for PMSM system using sliding-mode control and disturbance compensation techniques," IEEE Transactions on Power Electronics, vol. 28, no. 3, pp. 1358-1365, 2013.

[32] J. A. Farrell, M. Polycarpou, M. Sharma, and W. Dong, "Command filtered backstepping," IEEE Transactions on Automatic Control, vol. 54, no. 6, pp. 1391-1395, 2009.

[33] X. Shao and H. Wang, "Active disturbance rejection based trajectory linearization control for hypersonic reentry vehicle with bounded uncertainties," ISA Transactions, vol. 54, pp. 27-38, 2015. 


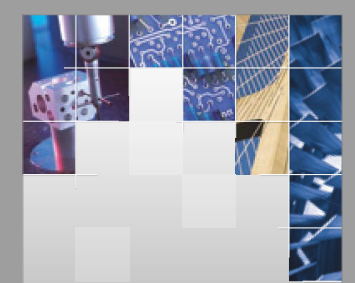

\section{Enfincering}
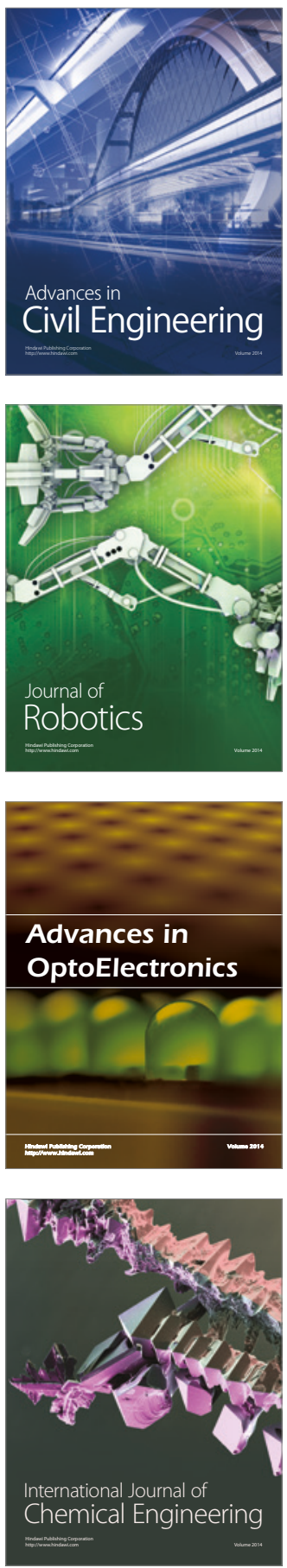

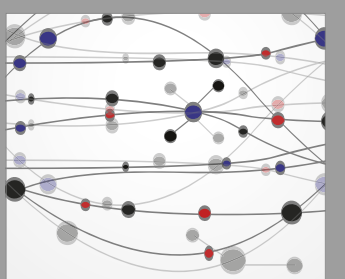

The Scientific World Journal

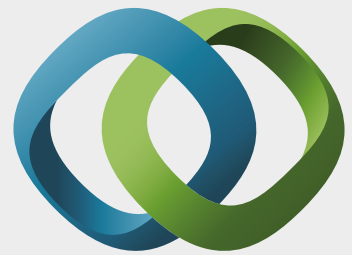

\section{Hindawi}

Submit your manuscripts at

https://www.hindawi.com
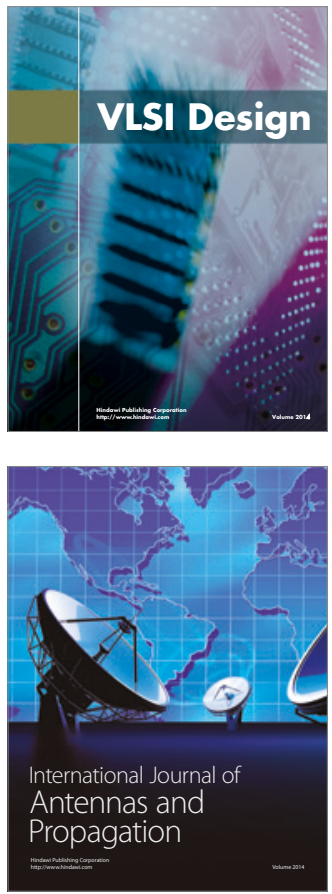

\section{Rotating}

Machinery
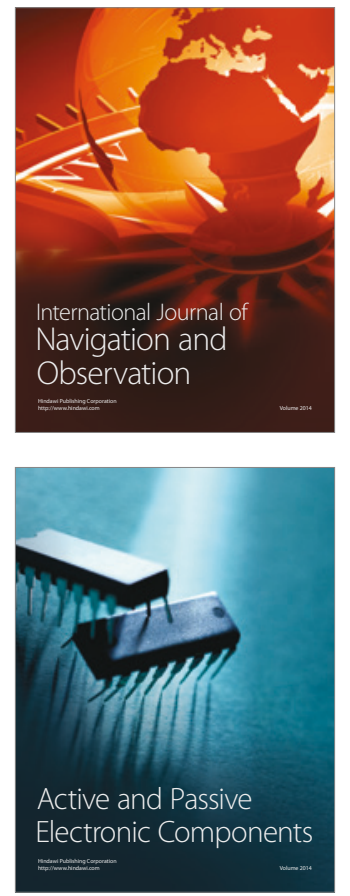
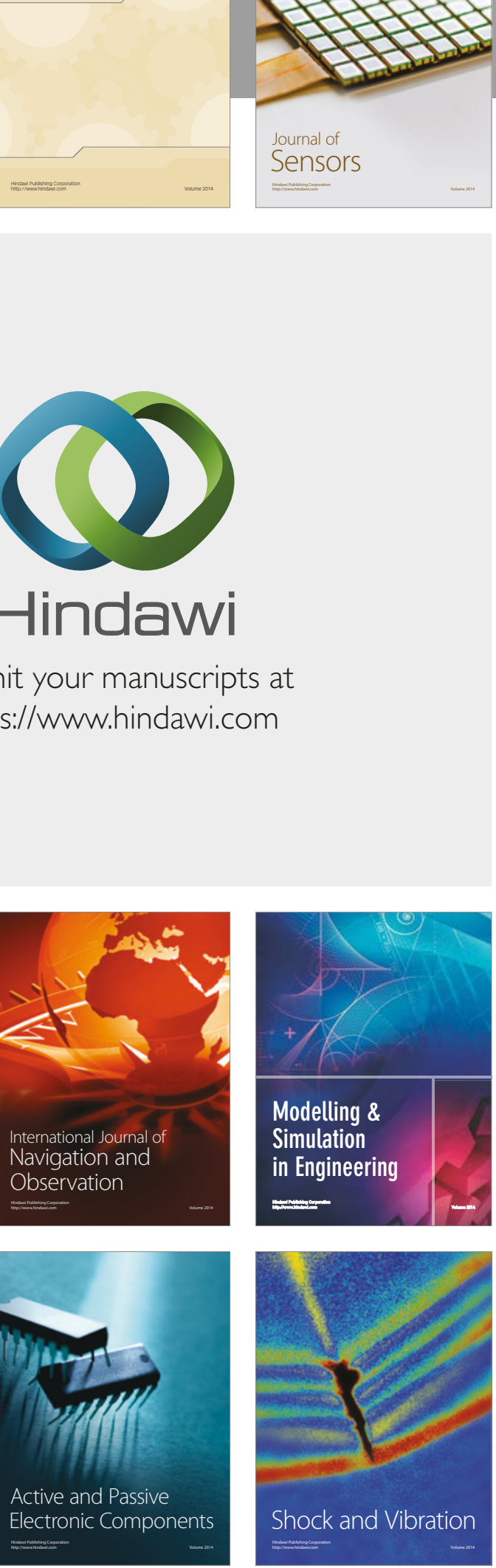
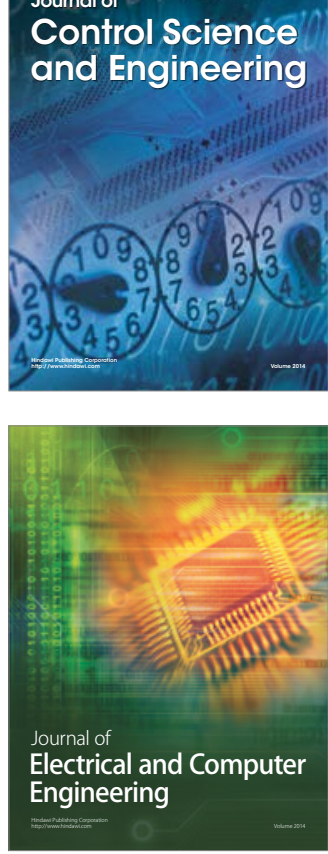

Distributed

Journal of

Control Science

and Engineering
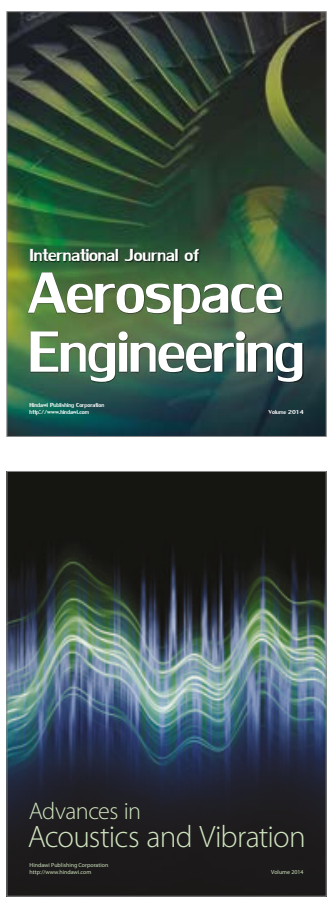

Sensor Networks 